\title{
Ortho-Phenylene-Bridged $\mathrm{Cp}$ /Amido Titanium Complexes for Ethylene/1-Hexene Copolymerization
}

Dae Joon Cho, ${ }^{a}$ Chun Ji Wu, ${ }^{a}$ Sujith $S,{ }^{a}$ Won-Sik Han, ${ }^{b}$ Sang Ook Kang, ${ }^{b}$ and Bun Yeoul Lee $*^{a}$

${ }^{a}$ Department of Molecular Science and Technology, Ajou University, Suwon 443-749 Korea and

${ }^{b}$ Department of Chemistry, Korea University, 208 Seochang, Chochiwon, Chung-nam, 339-700, Korea

Supporting Information 
General Remarks. All manipulations were performed under an inert atmosphere using standard glove box and Schlenk techniques. Toluene, pentane, hexane, THF, and $\mathrm{C}_{6} \mathrm{D}_{6}$ were distilled from benzophenone ketyl. Toluene used for polymerization reactions was purchased from Aldrich (anhydrous grade) and purified further over $\mathrm{Na} / \mathrm{K}$ alloy. Water used for the Suzuki-coupling was degassed by bubbling of nitrogen gas for 30 minutes. Dimethoxyethane (DME) used for the Suzuki-coupling was degassed by repeating the evacuation at $-78{ }^{\circ} \mathrm{C}$ and the warming to room temperature three times. Ethylene was purchased from Conley Gas $(99.9 \%)$ and purified by contacting with molecular sieves and copper. NMR spectra were recorded on a Varian Mercury plus 400 or Bruker spectrometer. Elemental analyses were carried out at the Inter-University Center Natural Science Facilities, Seoul National University. Gel permeation chromatograms (GPC) were obtained at $140{ }^{\circ} \mathrm{C}$ in trichlorobenzene using Waters Model 150-C+ GPC and the data were analyzed using a polystyrene analyzing curve. 3,4Dimethyl-2-cyclopenten-1-one, ${ }^{1}$ compound $\mathbf{4 f},{ }^{2} 4,4^{\prime}$-methylenebis(2-bromoaniline), ${ }^{3}$ and 4,4"-diamino$o$-terphenyl ${ }^{4}$ were prepared by the literature methods.

2-Bromo-3,4-dimethyl-2-cyclopenten-1-one (1). 3,4-Dimethyl-2-cyclopenten-1-one (56.0 g, 0.507 mol) was dissolved in $\mathrm{CH}_{2} \mathrm{Cl}_{2}(500 \mathrm{~mL})$ in $2.0 \mathrm{~L}$ three neck flask. The solution was cooled to $0{ }^{\circ} \mathrm{C}$ with an ice bath. Bromine (81.2 g, $0.507 \mathrm{~mol})$ dissolved in $\mathrm{CH}_{2} \mathrm{Cl}_{2}(500 \mathrm{~mL})$ was added dropwise for 2 hours while keeping the temperature below $5{ }^{\circ} \mathrm{C}$. The solution was stirred until its color became pale yellow from light red ( $\sim 2$ hours). Triethylamine $(61.7 \mathrm{~g}, 0.610 \mathrm{~mol})$ was added dropwise while keeping the temperature below $5{ }^{\circ} \mathrm{C}$. The solution was allowed to warm to room temperature and stirred overnight. Aqueous $\mathrm{HCl}$ solution $(1.0 \mathrm{~N}, 320 \mathrm{~mL})$ was added and the mixture was stirred for $30 \mathrm{~min}$. The mixture was transferred to a separatory funnel and the aqueous layer was removed. The organic layer was washed with saturated aqueous $\mathrm{NaHCO}_{3}$ solution $(300 \mathrm{~mL})$. Solvent was removed by rotary evaporator to give a residue which was dissolved in diethyl ether $(300 \mathrm{~mL})$. The etheral solution was washed with water $(300 \mathrm{~mL} \times 2)$ to remove any residual $\mathrm{HBr}$ salt of triethylamine. After the etheral solution was dried over anhydrous $\mathrm{MgSO}_{4}$, solvent was removed with rotary evaporator to give a residue, which was distilled under reduced pressure $\left(64{ }^{\circ} \mathrm{C} / 0.5\right.$ torr $)$ to give colorless oil $(60.1 \mathrm{~g}, 63 \%) .{ }^{1} \mathrm{H}$ NMR $\left(\mathrm{CDCl}_{3}\right)$ : $\delta 1.25\left(\mathrm{~d}, J=6.8 \mathrm{~Hz}, 3 \mathrm{H}, \mathrm{CH}_{3}\right), 2.15\left(\mathrm{dd}, J=18.8,8.8 \mathrm{~Hz}, 1 \mathrm{H}, \mathrm{CH}_{2}\right), 2.16\left(\mathrm{~s}, 3 \mathrm{H}, \mathrm{CH}_{3}\right), 2.78(\mathrm{dd}, J=$ 18.8, $\left.2.0 \mathrm{~Hz}, 1 \mathrm{H}, \mathrm{CH}_{2}\right), 2.87-2.94(\mathrm{~m}, 1 \mathrm{H}, \mathrm{CH}) \mathrm{ppm} .{ }^{13} \mathrm{C}\left\{{ }^{1} \mathrm{H}\right\} \mathrm{NMR}\left(\mathrm{CDCl}_{3}\right): \delta \quad 17.03,19.04,38.45$, 41.80, $122.82(\mathrm{C}=\mathrm{C}), 177.06(\mathrm{C}=\mathrm{C}), 200.15(\mathrm{C}=\mathrm{O}) \mathrm{ppm}$.

\section{2-Bromo-3,4-dimethyl-2-cyclopenten-1-one ethylene ketal (2). 2-Bromo-3,4-dimethyl-2-} cyclopenten-1-one $(60.0 \mathrm{~g}, 0.317 \mathrm{~mol})$, ethylene glycol $(138 \mathrm{~g}, 2.22 \mathrm{~mol})$, and triethyl orthoformate (142 g, $0.952 \mathrm{~mol})$ were mixed in $1.0 \mathrm{~L}$ flask. $p$-Toluenesulfonic acid monohydrate $(3.02 \mathrm{~g}, 15.9 \mathrm{mmol})$ was added and the solution was stirred overnight at room temperature. Saturated aqueous $\mathrm{NaHCO}_{3}(300$ 
$\mathrm{mL}$ ) was added and the product was extracted with hexane $(300 \mathrm{~mL} \times 4)$. The hexane phase was washed with water $(300 \mathrm{~mL} \times 3)$ and dried over anhydrous $\mathrm{MgSO}_{4}$. Solvent was removed by rotary evaporator to give a residue which was distilled under full vacuum (0.5 torr). Unreacted triethyl orthoformate was distilled off at $\sim 42{ }^{\circ} \mathrm{C}$ and the product was collected at $\sim 62{ }^{\circ} \mathrm{C}(57.0 \mathrm{~g}, 77 \%)$. In some cases, abrupt decomposition of the product to black shoot was observed during distillation. Addition of solid $\mathrm{K}_{2} \mathrm{CO}_{3}$ $(0.8 \mathrm{~g})$ to the distillation pot consistently blocked the decomposition. ${ }^{1} \mathrm{H}$ NMR $\left(\mathrm{CDCl}_{3}\right): \delta 1.13(\mathrm{~d}, J=$ $\left.6.8 \mathrm{~Hz}, 3 \mathrm{H}, \mathrm{CH}_{3}\right)$, 1.76. (dd, $\left.J=13.6,4.4 \mathrm{~Hz}, 1 \mathrm{H}, \mathrm{CH}_{2}\right), 1.79\left(\mathrm{~s}, 3 \mathrm{H}, \mathrm{CH}_{3}\right), 2.41$ (dd, $J=13.6,8.0 \mathrm{~Hz}$, $\left.1 \mathrm{H}, \mathrm{CH}_{2}\right), 2.60-2.68(\mathrm{~m}, 1 \mathrm{H}, \mathrm{CH}), 3.94-4.01\left(\mathrm{~m}, 2 \mathrm{H}, \mathrm{CH}_{2} \mathrm{O}\right), 4.16-4.21\left(\mathrm{~m}, 2 \mathrm{H}, \mathrm{CH}_{2} \mathrm{O}\right) \mathrm{ppm} .{ }^{13} \mathrm{C}\left\{{ }^{1} \mathrm{H}\right\}$ NMR $\left(\mathrm{CDCl}_{3}\right): \delta 14.14,19.11,38.74,42.91,65.24(\mathrm{C}-\mathrm{O}), 65.43(\mathrm{C}-\mathrm{O}), 116.37(\mathrm{C}=\mathrm{C}), 118.93(\mathrm{C}=\mathrm{C})$, 147.72 (OCO) ppm.

2-(Dihydroxyboryl)-3,4-dimethyl-2-cyclopenten-1-one (3). To a Schlenk flask containing 2-bromo3,4-dimethyl-2-cyclopenten-1-one ethylene ketal (57.3 g, $0.246 \mathrm{~mol})$ was added THF (300 ml). After the solution was cooled to $-78^{\circ} \mathrm{C}, \mathrm{n}-\mathrm{BuLi}(98.3 \mathrm{~mL}, 2.5 \mathrm{M}$ in hexane, $0.245 \mathrm{~mol})$ was added via syringe. The solution was stirred for 1 hour at $-78^{\circ} \mathrm{C}$. A cooled solution of $\mathrm{B}(\mathrm{OiPr})_{3}(50.9 \mathrm{~g}, 0.270 \mathrm{~mol})$ in THF $(50 \mathrm{ml})$ at $-78{ }^{\circ} \mathrm{C}$ was added to the flask containing the lithiated compound via a cannula. After the solution was stirred for 1.5 hour at $-78^{\circ} \mathrm{C}$, it was slowly warmed to $-30^{\circ} \mathrm{C}$ for 30 minutes. Aqueous $\mathrm{HCl}$ solution $(2 \mathrm{~N}, 300 \mathrm{~mL})$ was added and the reaction mixture was transferred to a separatory funnel containing ethyl acetate $(300 \mathrm{~mL})$. Organic phase was collected and the aqueous phase was extracted with additional ethyl acetate $(300 \mathrm{~mL})$. After the combined organic phase was dried over anhydrous $\mathrm{MgSO}_{4}$, solvent was removed with rotary evaporator to give a yellow residue, which was triturated in hexane to give a white solid. The solid was collected by filtration $(20.3 \mathrm{~g})$. The filtrate was collected and solvent was removed by rotary evaporator to give an oily residue to which aqueous $\mathrm{HCl}$ solution (2 $\mathrm{N}, 40 \mathrm{~mL})$ and hexane $(20 \mathrm{~mL})$ were added. Additional white solid was obtained by stirring the mixture for several hours. The solid was collected by filtration and washed with cold hexane (7.6 g). From the filtrate, organic phase was collected and stored in a freezer to give additional crops which was collected by decantation (3.6 g). The combined yield was $85 \%$ (31.5 g). M.p. $79{ }^{\circ} \mathrm{C}$. IR (neat): $3301(\mathrm{O}-\mathrm{H}), 1650$ $(\mathrm{C}=\mathrm{O}) \mathrm{cm}^{-1} .{ }^{1} \mathrm{H} \mathrm{NMR}\left(\mathrm{CDCl}_{3}\right): \delta 1.24\left(\mathrm{~d}, J=3.6 \mathrm{~Hz}, 3 \mathrm{H}, \mathrm{CH}_{3}\right), 2.09\left(\mathrm{dd}, J=19,2.0 \mathrm{~Hz}, 1 \mathrm{H}, \mathrm{CH}_{2}\right), 2.39$ (s, 3H, $\left.\mathrm{CH}_{3}\right), 2.72\left(\mathrm{dd}, J=19,6.8 \mathrm{~Hz}, 1 \mathrm{H}, \mathrm{CH}_{2}\right), 2.84-2.86(\mathrm{~m}, 1 \mathrm{H}, \mathrm{CH}), 7.29$ (s, 2H, OH) ppm. ${ }^{13} \mathrm{C}\left\{{ }^{1} \mathrm{H}\right\} \mathrm{NMR}\left(\mathrm{CDCl}_{3}\right): \delta 18.01,18.90,40.76,44.22,197.08,216.12$ ppm. Anal. Calc. $\left(\mathrm{C}_{7} \mathrm{H}_{11} \mathrm{BO}_{3}\right): \mathrm{C}$ : $54.60 ; \mathrm{H}, 7.20 \%$. Found: C, 54.40; H, $7.42 \%$.

Compound 4a. 2-Bromoaniline (1.65 g, $9.56 \mathrm{mmol})$ and cyclohexanone (4.693 g, $47.81 \mathrm{mmol})$ were dissolved in benzene $(9 \mathrm{ml})$ and molecular sieves (4 A, $2.0 \mathrm{~g}$ ) was added. The flask was connected with a Dean-Stark apparatus and the solution was refluxed for 4 days. After the solution was cooled to room 
temperature, the molecular sieves were filtered off. All volatiles were removed under vacuum at $60{ }^{\circ} \mathrm{C}$ to give a crude imine compound. The imine compound was dissolved in degassed methanol (28 $\mathrm{ml})$. Sodium borohydride $(1.08 \mathrm{~g}, 28.7 \mathrm{mmol})$ was added slowly under weak stream of nitrogen and the mixture was stirred at room temperature for 2 hours. Aqueous $1 \mathrm{~N} \mathrm{KOH}(20 \mathrm{~mL})$ was added and the product was extracted with methylene chloride $(30 \mathrm{~mL} \times 2)$. The combined organic layer was dried over $\mathrm{MgSO}_{4}$ and solvent was removed by rotary evaporator to give a residue which was purified by column chromatography on silica gel eluting with hexane. Colorless oil was obtained (1.43 g, $59 \%$ ). IR (neat): $3401(\mathrm{~N}-\mathrm{H}) \mathrm{cm}^{-1} .{ }^{1} \mathrm{H}$ NMR $\left(\mathrm{CDCl}_{3}\right): \delta 0.99(\mathrm{t}, J=\mathrm{Hz}, 2 \mathrm{H}, \mathrm{Cy}), 1.29-1.53$ (m, 3H, Cy), 1.72-1.75 (m, $1 \mathrm{H}, \mathrm{Cy}), 1.88-1.84$ (m, 2H, Cy), 2.11-2.14 (m, 2H, Cy), 3.36-3.40 (m, 1H, N-CH), 4.34 (br s, 1H, NH), $6.58\left(\mathrm{td}, J=7.6,0.8 \mathrm{~Hz}, 1 \mathrm{H}, \mathrm{C}_{6} \mathrm{H}_{4}\right), 6.71\left(\mathrm{~d}, J=7.6 \mathrm{~Hz}, 1 \mathrm{H}, \mathrm{C}_{6} \mathrm{H}_{4}\right), 7.20\left(\mathrm{td}, J=7.6,0.8 \mathrm{~Hz}, 1 \mathrm{H}, \mathrm{C}_{6} \mathrm{H}_{4}\right)$, $7.47\left(\mathrm{dd}, J=7.6,1.2 \mathrm{~Hz}, 1 \mathrm{H}, \mathrm{C}_{6} \mathrm{H}_{4}\right) \mathrm{ppm} .{ }^{13} \mathrm{C}\left\{{ }^{1} \mathrm{H}\right\} \operatorname{NMR}\left(\mathrm{CDCl}_{3}\right): \delta 24.91,25.94,33.13,51.53,109.62$, 111.58, 116.92, 128.16, 132.33, 143.83 ppm. Anal. Calc. $\left(\mathrm{C}_{12} \mathrm{H}_{16} \mathrm{BrN}\right)$ : C, 56.71; H, 6.35; N, $5.51 \%$. Found: C, 56.67; H, 6.58; N, $5.82 \%$.

$N$-Cyclohexyl-4-methylaniline. $p$-Toluidine $(3.85 \mathrm{~g}, 35.9 \mathrm{mmol})$ and cyclohexanone $(21.2 \mathrm{~g}, 0.216$ mol) were dissolved in toluene $(25 \mathrm{ml})$ and molecular sieves $(4 \mathrm{~A}, 7.0 \mathrm{~g})$ was added. The flask was sealed with screw-cap and heated at $100{ }^{\circ} \mathrm{C}$ for 2 days. After the molecular sieves were filtered off, all volatiles were removed under vacuum at $60{ }^{\circ} \mathrm{C}$ to give a crude imine compound. The imine compound was reduced with sodium borohydride $(4.08 \mathrm{~g}, 108 \mathrm{mmol})$ by the same conditions and procedures as for 4a. The product was purified by column chromatography on silica eluting with hexane and ethyl acetate (10 : 1). A white solid was obtained (5.16 g, 76 \%). M.p. $39{ }^{\circ} \mathrm{C}$. IR (neat): $3460(\mathrm{~N}-\mathrm{H}) \mathrm{cm}^{-1}$. ${ }^{1} \mathrm{H}$ NMR $\left(\mathrm{C}_{6} \mathrm{D}_{6}\right): \delta$ 0.88-0.94 (m, 2H, Cy), 1.04-1.09 (m, 1H, Cy), 1.12-1.21 (m, 2H, Cy), 1.46-1.50 (m, 1H, Cy), 1.55-1.60 (m, 2H, Cy), 1.90-1.93 (m, 2H, Cy), 2.23 (s, 3H, $\left.\mathrm{CH}_{3}\right), 3.03$ (br s, 1H, NH), 3.04-3.10 (m, 1H, $\mathrm{N}-\mathrm{CH}), 6.45\left(\mathrm{~d}, J=8.0 \mathrm{~Hz}, 2 \mathrm{H}, \mathrm{C}_{6} \mathrm{H}_{4}\right), 7.00\left(\mathrm{~d}, J=8.0 \mathrm{~Hz}, 2 \mathrm{H}, \mathrm{C}_{6} \mathrm{H}_{4}\right) \mathrm{ppm} .{ }^{13} \mathrm{C}\left\{{ }^{1} \mathrm{H}\right\} \mathrm{NMR}\left(\mathrm{C}_{6} \mathrm{D}_{6}\right): \delta$ $20.85,25.50,26.50,33.82,52.04,113.82,125.78,130.03,145.60$ ppm. Anal. Calc. $\left(\mathrm{C}_{13} \mathrm{H}_{19} \mathrm{~N}\right): \mathrm{C}$, 82.48; H, 10.12; N, 7.40 \%. Found: C, 82.26; H, 10.27; N, 7.19\%.

Compound 4b. $N$-Cyclohexyl-4-methylaniline $(2.00 \mathrm{~g}, 10.6 \mathrm{mmol})$ was dissolved in methylene chloride $(20 \mathrm{~mL})$ and $\mathrm{Br}_{2}(1.69 \mathrm{~g}, 10.6 \mathrm{mmol})$ in methylene chloride $(16 \mathrm{ml})$ was added at $0{ }^{\circ} \mathrm{C}$ for 30 minutes. The solution was stirred further for 2 hours. After an aqueous $1 \mathrm{~N} \mathrm{KOH}(20 \mathrm{ml})$ was added, the product was extracted with methylene chloride $(40 \mathrm{~mL} \times 2)$. The combined organic layer was dried over $\mathrm{MgSO}_{4}$ and solvent was removed by rotary evaporator to give an residue which was purified by column chromatography on silica gel eluting with hexane and ethyl acetate $(10: 1)$. Colorless oil was obtained (2.67 g, 94 \%). IR (neat): $3401(\mathrm{~N}-\mathrm{H}) \mathrm{cm}^{-1} .{ }^{1} \mathrm{H}$ NMR $\left(\mathrm{C}_{6} \mathrm{D}_{6}\right): \delta$ 0.98-1.03 (m, 3H, Cy), 1.10-1.16 (m, 3H, Cy), 1.39-1.42 (m, 1H, Cy), 1.53-1.56 (m, 2H, Cy), 1.82-1.85 (m, 2H, Cy), 2.03 (s, 3H, CH $)$, 3.06-3.08 (m, 1H, N-CH), 4.16 (br d, $J=7.2 \mathrm{~Hz}, 1 \mathrm{H}, \mathrm{NH}), 6.46$ (d, $\left.J=7.6 \mathrm{~Hz}, 1 \mathrm{H}, \mathrm{C}_{6} \mathrm{H}_{3}\right), 6.84$ (dd, $J=1.6,7.6$ 
$\left.\mathrm{Hz}, 1 \mathrm{H}, \mathrm{C}_{6} \mathrm{H}_{3}\right), 7.23\left(\mathrm{~d}, J=1.6 \mathrm{~Hz}, 1 \mathrm{H}, \mathrm{C}_{6} \mathrm{H}_{3}\right) \mathrm{ppm} .{ }^{13} \mathrm{C}\left\{{ }^{1} \mathrm{H}\right\} \mathrm{NMR}\left(\mathrm{C}_{6} \mathrm{D}_{6}\right): \delta 20.25,25.21,26.29,33.37$, 51.90, 110.25, 112.29, 126.71, 129.24, 133.29, 142.34 ppm. Anal. Calc. $\left(\mathrm{C}_{13} \mathrm{H}_{18} \mathrm{BrN}\right): \mathrm{C}, 58.22 ; \mathrm{H}$, 6.76 ; N, $5.22 \%$. Found: C, 58.05; H, 6.84; N, $4.96 \%$.

$N$-Cyclohexyl-4-phenylaniline. The compound was prepared by the same conditions and procedures as for $N$-cyclohexyl-4-methylaniline using benzidine. A light yellow solid was obtained (65 \%). M.p. 75 ${ }^{\circ} \mathrm{C}$. IR (neat): $3385(\mathrm{~N}-\mathrm{H}) \mathrm{cm}^{-1} .{ }^{1} \mathrm{H}$ NMR $\left(\mathrm{C}_{6} \mathrm{D}_{6}\right): \delta$ 0.84-0.94 (m, 2H, Cy), 1.02-1.21 (m, 3H, Cy), 1.461.59 (m, 3H, Cy), 1.88-1.91 (m, 2H, Cy), 3.06-3.11 (m, 1H, N-CH), 3.18 (br s, 1H, NH), 6.49 (d, J=8.0 $\left.\mathrm{Hz}, 2 \mathrm{H}, \mathrm{C}_{6} \mathrm{H}_{4}\right), 7.14\left(\mathrm{t}, J=8.0 \mathrm{~Hz}, 1 \mathrm{H}, \mathrm{C}_{6} \mathrm{H}_{5}\right), 7.28\left(\mathrm{t}, J=8.0 \mathrm{~Hz}, 2 \mathrm{H}, \mathrm{C}_{6} \mathrm{H}_{5}\right), 7.49(\mathrm{~d}, J=8.0 \mathrm{~Hz}, 2 \mathrm{H}$, $\left.\mathrm{C}_{6} \mathrm{H}_{4}\right), 7.60\left(\mathrm{~d}, J=8.0 \mathrm{~Hz}, 2 \mathrm{H}, \mathrm{C}_{6} \mathrm{H}_{5}\right) \mathrm{ppm} .{ }^{13} \mathrm{C}\left\{{ }^{1} \mathrm{H}\right\} \mathrm{NMR}\left(\mathrm{C}_{6} \mathrm{D}_{6}\right): \delta 25.43,26.41,33.66,51.70,113.78$, 126.15, 126.59, 128.27, 129.00, 130.10, 142.03, 147.14 ppm. Anal. Calc. $\left(\mathrm{C}_{18} \mathrm{H}_{21} \mathrm{~N}\right)$ : C, 86.01; H, 8.42; N, $5.57 \%$. Found: C, 85.95; H, 8.26; N, $5.32 \%$.

Compound 4c. The compound was synthesized by the same conditions and procedures as for $\mathbf{4 b}$ using $N$-cyclohexyl-4-phenylaniline. It was purified by column chromatography on silica gel eluting with hexane and methylene chloride (4 : 1). Colorless oil was obtained (73 \%). IR (neat): 3401 (N-H) $\mathrm{cm}^{-1} .{ }^{1} \mathrm{H}$ NMR $\left(\mathrm{C}_{6} \mathrm{D}_{6}\right): \delta$ 0.96-1.05 (m, 2H, Cy), 1.06-1.18 (m, 2H, Cy), 1.23-1.31 (m, 1H, Cy), 1.391.44 (m, 1H, Cy), 1.52-1.56 (m, 2H, Cy), 1.79-1.83 (m, 2H, Cy), 3.02-3.11 (m, 1H, N-CH), 4.35 (br d, J $=7.2 \mathrm{~Hz}, 1 \mathrm{H}, \mathrm{NH}), 6.52\left(\mathrm{~d}, J=8.0 \mathrm{~Hz}, 1 \mathrm{H}, \mathrm{C}_{6} \mathrm{H}_{3}\right), 7.11\left(\mathrm{tt}, J=1.6,8.0 \mathrm{~Hz}, 1 \mathrm{H}, \mathrm{C}_{6} \mathrm{H}_{5}\right), 7.21(\mathrm{t}, J=8.0$ $\left.\mathrm{Hz}, 2 \mathrm{H}, \mathrm{C}_{6} \mathrm{H}_{5}\right), 7.34\left(\mathrm{dd}, J=2.4,8.4 \mathrm{~Hz}, 1 \mathrm{H}, \mathrm{C}_{6} \mathrm{H}_{3}\right), 7.37\left(\mathrm{dd}, J=1.2,8.0 \mathrm{~Hz}, 2 \mathrm{H}, \mathrm{C}_{6} \mathrm{H}_{5}\right), 7.78(\mathrm{~d}, J=$ $\left.2.4 \mathrm{~Hz}, 1 \mathrm{H}, \mathrm{C}_{6} \mathrm{H}_{3}\right)$ ppm. ${ }^{13} \mathrm{C}\left\{{ }^{1} \mathrm{H}\right\} \operatorname{NMR}\left(\mathrm{C}_{6} \mathrm{D}_{6}\right): \delta 25.13,26.20,33.22,51.70,110.72,112.28,126.56$, 126.62, 127.36, 129.02, 130.93, 131.42, 140.42, 143.67 ppm. Anal. Calc. $\left(\mathrm{C}_{18} \mathrm{H}_{20} \mathrm{BrN}\right)$ : C, 65.46; H, $6.10 ; \mathrm{N}, 4.24$ \%. Found: C, 65.29; H, 5.85; N, $4.36 \%$.

4-Chloro- $N$-cyclohexylaniline. The compound was prepared by the same conditions and procedures as for $\mathrm{N}$-cyclohexyl-4-methylaniline using 4-chloroaniline. It was purified by column chromatography on silica gel eluting with hexane and ethyl acetate (10:1). A white solid was obtained (71 \%). M.p. 41 ${ }^{\circ} \mathrm{C}$. IR (neat): $3408(\mathrm{~N}-\mathrm{H}) \mathrm{cm}^{-1} .{ }^{1} \mathrm{H}$ NMR $\left(\mathrm{C}_{6} \mathrm{D}_{6}\right): \delta$ 0.77-0.85 (m, 2H, Cy), 1.01-1.17 (m, 3H, Cy), $1.45-$ 1.56 (m, 3H, Cy), 1.76-1.79 (m, 2H, Cy), 2.85-2.90 (m, 1H, N-CH), 3.03 (br s, 1H, NH), 6.15 (d, J=8.8 $\left.\mathrm{Hz}, 2 \mathrm{H}, \mathrm{C}_{6} \mathrm{H}_{4}\right), 7.09\left(\mathrm{~d}, J=8.8 \mathrm{~Hz}, 2 \mathrm{H}, \mathrm{C}_{6} \mathrm{H}_{4}\right) \mathrm{ppm} .{ }^{13} \mathrm{C}\left\{{ }^{1} \mathrm{H}\right\} \mathrm{NMR}\left(\mathrm{C}_{6} \mathrm{D}_{6}\right): \delta 25.36,26.34,33.44,51.70$, 114.42, 121.31, 129.30, 146.21 ppm. Anal. Calc. $\left(\mathrm{C}_{12} \mathrm{H}_{16} \mathrm{ClN}\right)$ : C, 68.73; H, 7.69; N, 6.68 \%. Found: C, $68.52 ; \mathrm{H}, 7.37$; N, $6.84 \%$.

Compound 4d. The compound was synthesized by the same conditions and procedures as for $\mathbf{4 b}$ using 4-chloro- $N$-cyclohexylaniline. It was purified by column chromatography on silica gel eluting with hexane and ethyl acetate $(10: 1)$. Colorless oil was obtained $(90 \%)$. IR (neat): $3401(\mathrm{~N}-\mathrm{H}) \mathrm{cm}^{-1}$. ${ }^{1} \mathrm{H}$ NMR $\left(\mathrm{C}_{6} \mathrm{D}_{6}\right): \delta$ 0.82-0.93 (m, 2H, Cy), 0.96-0.98 (m, 1H, Cy), 1.00-1.11 (m, 2H, Cy), 1.37-1.41 (m, 
1H, Cy), 1.36-1.41 (m, 2H, Cy), 1.66-1.69 (m, 2H, Cy), 2.79-2.90 (m, 1H, N-CH), 4.14 (br d, J = 7.2 Hz, $1 \mathrm{H}, \mathrm{NH}), 6.16\left(\mathrm{~d}, J=8.8 \mathrm{~Hz}, 1 \mathrm{H}, \mathrm{C}_{6} \mathrm{H}_{3}\right), 6.98\left(\mathrm{dd}, J=2.8,8.8 \mathrm{~Hz}, 1 \mathrm{H}, \mathrm{C}_{6} \mathrm{H}_{3}\right), 7.38(\mathrm{~d}, J=2.8 \mathrm{~Hz}, 1 \mathrm{H}$, $\left.\mathrm{C}_{6} \mathrm{H}_{3}\right)$ ppm. ${ }^{13} \mathrm{C}\left\{{ }^{1} \mathrm{H}\right\} \operatorname{NMR}\left(\mathrm{C}_{6} \mathrm{D}_{6}\right): \delta 25.06,26.15,33.03,51.68,109.87,112.42,121.04,128.57,132.16$, 143.12 ppm. Anal. Calc. $\left(\mathrm{C}_{12} \mathrm{H}_{15} \mathrm{BrClN}\right)$ : C, 49.94; H, 5.24; N, 4.85 \%. Found: $\mathrm{C}, 50.12$; H, 5.39; N, $4.94 \%$.

$\mathrm{N}$-Cyclohexyl-4-fluoroaniline. The compound was prepared by the same conditions and procedures as for $N$-cyclohexyl-4-methylaniline using 4-fluoroaniline. It was purified by column chromatography on silica gel eluting with hexane and ethyl acetate $(50: 1)$. Brown oil was obtained (92\%). IR (neat): $3413(\mathrm{~N}-\mathrm{H}) \mathrm{cm}^{-1} .{ }^{1} \mathrm{H}$ NMR $\left(\mathrm{C}_{6} \mathrm{D}_{6}\right): \delta$ 0.83-0.92 (m, 2H, Cy), 1.00-1.22 (m, 3H, Cy), 1.47-1.52 (m, 1H, Cy), 1.56-1.60 (m, 2H, Cy), 1.82-1.85 (m, 2H, Cy), 2.89-2.95 (m, 1H, N-CH), 3.00 (br s, 1H, NH), 6.22 $\left(\mathrm{dd}, J=4.4,8.8 \mathrm{~Hz}, 2 \mathrm{H}, \mathrm{C}_{6} \mathrm{H}_{4}\right), 6.79\left(\mathrm{t}, J=8.8 \mathrm{~Hz}, 2 \mathrm{H}, \mathrm{C}_{6} \mathrm{H}_{4}\right) \mathrm{ppm} .{ }^{13} \mathrm{C}\left\{{ }^{1} \mathrm{H}\right\} \operatorname{NMR}\left(\mathrm{C}_{6} \mathrm{D}_{6}\right): \delta 25.45$, $26.43,33.64,52.33,114.13\left(\mathrm{~d},{ }^{3} J_{\mathrm{CF}}=6.8 \mathrm{~Hz}, \mathrm{C}_{6} \mathrm{H}_{4} \mathrm{~F}\right), 155.78\left(\mathrm{~d},{ }^{2} J_{\mathrm{CF}}=22 \mathrm{~Hz}, \mathrm{C}_{6} \mathrm{H}_{4} \mathrm{~F}\right), 144.18\left(\mathrm{~d},{ }^{4} J_{\mathrm{CF}}\right.$ $\left.=1.5 \mathrm{~Hz}, \mathrm{C}_{6} \mathrm{H}_{4} \mathrm{~F}-\mathrm{C}\right), 155.66$ (d, $\left.{ }^{1} J_{\mathrm{CF}}=231.3 \mathrm{~Hz}, \mathrm{C}_{6} \mathrm{H}_{4} \mathrm{~F}-\mathrm{C}\right)$ ppm. Anal. Calc. $\left(\mathrm{C}_{12} \mathrm{H}_{16} \mathrm{FN}\right): \mathrm{C}, 74.58 ; \mathrm{H}$, 8.34 ; N, $7.25 \%$. Found: C, 74.35; H, 8.27; N, $7.42 \%$.

Compound 4e. The compound was synthesized by the same conditions and procedures as for $\mathbf{4 b}$ using $N$-cyclohexyl-4-fluoroaniline. It was purified by column chromatography on silica gel eluting with hexane and ethyl acetate $(50: 1)$. Colorless oil was obtained $(92 \%)$. IR (neat): $3401(\mathrm{~N}-\mathrm{H}) \mathrm{cm}^{-1}$. ${ }^{1} \mathrm{H}$ NMR $\left(\mathrm{C}_{6} \mathrm{D}_{6}\right): \delta$ 0.90-0.99 (m, 2H, Cy), 1.03-1.19 (m, 3H, Cy), 1.42-1.46 (m, 1H, Cy), 1.53-1.57 (m, 2H, Cy), 1.75-1.78 (m, 2H, Cy), 2.87-2.96 (m, 1H, N-CH), 3.97 (d, $J=7.2 \mathrm{~Hz}, 1 \mathrm{H}, \mathrm{NH}), 6.23$ (dd, $J=$ $\left.4.4,8.8 \mathrm{~Hz}, 1 \mathrm{H}, \mathrm{C}_{6} \mathrm{H}_{3}\right), 6.72\left(\mathrm{td}, J=2.8,8.8 \mathrm{~Hz}, 1 \mathrm{H}, \mathrm{C}_{6} \mathrm{H}_{3}\right), 7.09\left(\mathrm{dd}, J=2.8,8.0 \mathrm{~Hz}, 1 \mathrm{H}, \mathrm{C}_{6} \mathrm{H}_{3}\right) \mathrm{ppm}$. ${ }^{13} \mathrm{C}\left\{{ }^{1} \mathrm{H}\right\} \operatorname{NMR}\left(\mathrm{C}_{6} \mathrm{D}_{6}\right): \delta 25.20,26.28,33.29,52.15,109.14\left(\mathrm{~d},{ }^{3} J_{\mathrm{CF}}=9.9 \mathrm{~Hz}, \mathrm{C}_{6} \mathrm{H}_{3}\right), 111.99\left(\mathrm{~d},{ }^{3} J_{\mathrm{CF}}=\right.$ $\left.7.6 \mathrm{~Hz}, \mathrm{C}_{6} \mathrm{H}_{3}\right), 115.21\left(\mathrm{~d},{ }^{2} J_{\mathrm{CF}}=21.2 \mathrm{~Hz}, \mathrm{C}_{6} \mathrm{H}_{3}\right), 119.71\left(\mathrm{~d},{ }^{2} J_{\mathrm{CF}}=25 \mathrm{~Hz}, \mathrm{C}_{6} \mathrm{H}_{3}\right), 141.26\left(\mathrm{~d},{ }^{4} J_{\mathrm{CF}}=2.3\right.$ $\left.\mathrm{Hz}, \mathrm{C}_{6} \mathrm{H}_{3}\right), 154.32$ (d, $\left.J_{\mathrm{CF}}=236 \mathrm{~Hz}, \mathrm{C}_{6} \mathrm{H}_{3}\right)$ ppm. Anal. Calc. $\left(\mathrm{C}_{12} \mathrm{H}_{15} \mathrm{BrFN}\right): \mathrm{C}, 52.96 ; \mathrm{H}, 5.56 ; \mathrm{N}$, $5.15 \%$. Found: C, 53.15; H, 5.92; N, $5.37 \%$.

Compound 4g. The compound was prepared by the same conditions and procedures as for $\mathbf{4 a}$ using 2-bromoaniline and 3-pentanone. It was purified by column chromatography on silica gel eluting with hexane. Colorless oil was obtained (61\%). IR (neat): $3407(\mathrm{~N}-\mathrm{H}) \mathrm{cm}^{-1} .{ }^{1} \mathrm{H}$ NMR $\left(\mathrm{CDCl}_{3}\right): \delta 1.01(\mathrm{t}, J=$ $7.6 \mathrm{~Hz}, 6 \mathrm{H}, \mathrm{CH}_{3}$ ), 1.54-1.74 (m, 4H, $\mathrm{CH}_{2}$ ), 3.34 (quintet, $\left.J=6.0 \mathrm{~Hz}, 1 \mathrm{H}, \mathrm{N}-\mathrm{CH}\right), 4.25$ (s, 1H, NH), 6.56 $\left(\mathrm{td}, J=7.6,1.2 \mathrm{~Hz}, 1 \mathrm{H}, \mathrm{C}_{6} \mathrm{H}_{4}\right), 6.67\left(\mathrm{dd}, J=8.0,1.2 \mathrm{~Hz}, 1 \mathrm{H}, \mathrm{C}_{6} \mathrm{H}_{4}\right), 7.18\left(\mathrm{td}, J=7.6,1.6 \mathrm{~Hz}, 1 \mathrm{H}, \mathrm{C}_{6} \mathrm{H}_{4}\right)$, $7.45\left(\mathrm{dd}, J=8.0,1.6 \mathrm{~Hz}, 1 \mathrm{H}, \mathrm{C}_{6} \mathrm{H}_{4}\right) \mathrm{ppm} .{ }^{13} \mathrm{C}\left\{{ }^{1} \mathrm{H}\right\} \mathrm{NMR}\left(\mathrm{CDCl}_{3}\right): \delta 10.22,26.73,55.60,109.65,111.55$, 116.74, 128.19, 132.30, 144.54 ppm. Anal. Calc. $\left(\mathrm{C}_{11} \mathrm{H}_{16} \mathrm{BrN}\right)$ : C, 54.56; H, 6.66; N, 5.78 \%. Found: C, $54.36 ; \mathrm{H}, 6.48 ; \mathrm{N}, 5.48 \%$. 
Compound 5a. Compound 3 (1.27 g, $8.26 \mathrm{mmol}), \mathrm{Na}_{2} \mathrm{CO}_{3}(1.25 \mathrm{~g}, 11.8 \mathrm{mmol}), \mathrm{Pd}\left(\mathrm{PPh}_{3}\right)_{4}(0.182 \mathrm{~g}$, $0.157 \mathrm{mmol})$ and $4 \mathrm{a}(2.00 \mathrm{~g}, 7.87 \mathrm{mmol})$ were added into a Schlenk flask inside a glovebox. The flask was brought out and degassed DME $(21 \mathrm{~mL})$ and degassed water $(7 \mathrm{~mL})$ were added. The flask was sealed with screw-cap and heated at $95{ }^{\circ} \mathrm{C}$ overnight. The solution was cooled to room temperature and the product was extracted with ethyl acetate $(50 \mathrm{ml} \times 2)$. The combined organic layer was dried over anhydrous $\mathrm{MgSO}_{4}$ and solvent was removed by rotary evaporator to give a residue which was purified by column chromatography eluting with hexane and ethyl acetate $(5: 1)$. Yellow oil was obtained $(2.06$ g, 92 \%). IR (neat): $3379(\mathrm{~N}-\mathrm{H}), 1697(\mathrm{C}=\mathrm{O}) \mathrm{cm}^{-1} .{ }^{1} \mathrm{H}$ NMR $\left(\mathrm{CDCl}_{3}\right): \delta$ 1.13-1.28 (m, 4H, Cy), $1.32(\mathrm{~d}$, $\left.J=6.8 \mathrm{~Hz}, 3 \mathrm{H}, \mathrm{CH}_{3}\right), 1.35-1.41$ (m, 2H, Cy), 1.62-1.65 (m, 1H, Cy), 1.71-1.75 (m, 2H, Cy), 2.03 (s, 3H, $\left.\mathrm{CH}_{3}\right), 1.98-2.07(\mathrm{~m}, 1 \mathrm{H}, \mathrm{Cy}), 2.19\left(\mathrm{~d}, J=18.4 \mathrm{~Hz}, 1 \mathrm{H}, \mathrm{CH}_{2}\right), 2.83\left(\mathrm{dd}, J=18.8,6.8 \mathrm{~Hz}, 1 \mathrm{H}, \mathrm{CH}_{2}\right), 2.95$ (quintet, $J=6.8 \mathrm{~Hz}, 1 \mathrm{H}, \mathrm{CH}), 3.24-3.29(\mathrm{~m}, 1 \mathrm{H}, \mathrm{N}-\mathrm{CH}), 3.48(\mathrm{~s}, 1 \mathrm{H}, \mathrm{NH}), 6.71(\mathrm{t}, J=8.8 \mathrm{~Hz}, 1 \mathrm{H}$, $\left.\mathrm{C}_{6} \mathrm{H}_{4}\right), 6.74\left(\mathrm{~d}, J=8.8 \mathrm{~Hz}, 1 \mathrm{H}, \mathrm{C}_{6} \mathrm{H}_{4}\right), 6.88\left(\mathrm{~d}, J=8.8 \mathrm{~Hz}, 1 \mathrm{H}, \mathrm{C}_{6} \mathrm{H}_{4}\right), 7.20\left(\mathrm{t}, J=8.8 \mathrm{~Hz}, 1 \mathrm{H}, \mathrm{C}_{6} \mathrm{H}_{4}\right)$ ppm. ${ }^{13} \mathrm{C}\left\{{ }^{1} \mathrm{H}\right\} \mathrm{NMR}\left(\mathrm{CDCl}_{3}\right): \delta 16.31,19.54,24.82,25.88,33.13,37.59,43.52,51.43,111.34,116.13$, 117.19, 128.89, 129.44, 130.39, 144.72, 178.62, 206.65 ppm. Anal. Calc. $\left(\mathrm{C}_{19} \mathrm{H}_{25} \mathrm{NO}\right)$ : C, 80.52; $\mathrm{H}$, 8.89; N, $4.94 \%$. Found: C, 80.68; H, 9.05; N, $5.12 \%$.

Compound 5b. The compound was synthesized by the same conditions and procedures as for 5a using $\mathbf{4 b}$. It was purified by column chromatography on silica gel eluting with hexane and ethyl acetate (3 : 1). Yellow oil was obtained (98\%). IR (neat): $3378(\mathrm{~N}-\mathrm{H}), 1697(\mathrm{C}=\mathrm{O}) \mathrm{cm}^{-1} .{ }^{1} \mathrm{H}$ NMR $\left(\mathrm{C}_{6} \mathrm{D}_{6}\right): \delta$ 0.77 (d, $\left.J=6.8 \mathrm{~Hz}, 3 \mathrm{H}, \mathrm{CH}_{3}\right), 1.10-1.22$ (m, 4H, Cy), 1.42-1.48 (m, 2H, Cy), 1.54-1.62 (m, 2H, Cy), $1.63\left(\mathrm{~s}, 3 \mathrm{H}, \mathrm{CH}_{3}\right), 1.86$ (dd, $\left.J=2.4,18.4 \mathrm{~Hz}, 1 \mathrm{H}, \mathrm{CH}_{2}\right), 1.96-2.06(\mathrm{~m}, 2 \mathrm{H}, \mathrm{Cy}), 2.18-2.23(\mathrm{~m}, 1 \mathrm{H}, \mathrm{CH})$, 2.25 (s, 3H, $\left.\mathrm{C}_{6} \mathrm{H}_{3}-\mathrm{CH}_{3}\right), 2.46\left(\mathrm{dd}, J=6.8,18.4 \mathrm{~Hz}, 1 \mathrm{H}, \mathrm{CH}_{2}\right), 3.14-3.24$ (m, 1H, N-CH), 3.84 (br s, $1 \mathrm{H}$, $\mathrm{NH}), 6.73\left(\mathrm{~d}, J=8.4 \mathrm{~Hz}, 1 \mathrm{H}, \mathrm{C}_{6} \mathrm{H}_{3}\right), 6.86\left(\mathrm{br} \mathrm{s}, 1 \mathrm{H}, \mathrm{C}_{6} \mathrm{H}_{3}\right), 7.08\left(\mathrm{dd}, J=2.4,8.4 \mathrm{~Hz}, 1 \mathrm{H}, \mathrm{C}_{6} \mathrm{H}_{3}\right) \mathrm{ppm}$. ${ }^{13} \mathrm{C}\left\{{ }^{1} \mathrm{H}\right\} \operatorname{NMR}\left(\mathrm{C}_{6} \mathrm{D}_{6}\right): \delta 16.19,19.50,20.81,25.32,26.53,33.75,37.67,43.78,52.02,112.29,119.07$, 125.25, 129.85, 131.90, 139.93, 143.96, 176.77, 205.26 ppm. Anal. Calc. $\left(\mathrm{C}_{20} \mathrm{H}_{27} \mathrm{NO}\right): \mathrm{C}, 80.76$; $\mathrm{H}$, 9.15 ; N, $4.71 \%$. Found: C, 80.86; H, 8.89; N, $4.52 \%$.

Compound 5c. The compound was synthesized by the same conditions and procedures as for 5a using 4c. It was purified by column chromatography on silica gel eluting with hexane and ethyl acetate (3 : 1). Light yellow solid was obtained (98\%). M.p. $51-52{ }^{\circ} \mathrm{C}$, IR (neat): $3444(\mathrm{~N}-\mathrm{H}), 1637(\mathrm{C}=\mathrm{O}) \mathrm{cm}^{-1}$. ${ }^{1} \mathrm{H}$ NMR $\left(\mathrm{C}_{6} \mathrm{D}_{6}\right): \delta 0.75\left(\mathrm{~d}, J=6.8 \mathrm{~Hz}, 3 \mathrm{H}, \mathrm{CH}_{3}\right), 1.11-1.25(\mathrm{~m}, 4 \mathrm{H}, \mathrm{Cy}), 1.42-1.47$ (m, 2H, Cy), 1.561.62 (m, 2H, Cy), 1.61 (s, 3H, CH $\mathrm{CH}_{3}, 1.87$ (dd, $\left.J=2.0,18.4 \mathrm{~Hz}, 1 \mathrm{H}, \mathrm{CH}_{2}\right), 1.97-2.06$ (m, 2H, Cy), 2.16$2.26(\mathrm{~m}, 1 \mathrm{H}, \mathrm{CH}), 2.46\left(\mathrm{dd}, J=6.4,18.4 \mathrm{~Hz}, 1 \mathrm{H}, \mathrm{CH}_{2}\right), 3.17-3.29(\mathrm{~m}, 1 \mathrm{H}, \mathrm{N}-\mathrm{CH}), 4.14$ (br s, $\left.1 \mathrm{H}, \mathrm{NH}\right)$, $6.79\left(\mathrm{~d}, J=8.0 \mathrm{~Hz}, 1 \mathrm{H}, \mathrm{C}_{6} \mathrm{H}_{3}\right), 7.14\left(\mathrm{tt}, J=1.2,7.2 \mathrm{~Hz}, 1 \mathrm{H}, \mathrm{C}_{6} \mathrm{H}_{5}\right), 7.27$ (t, $\left.J=8.0 \mathrm{~Hz}, 2 \mathrm{H}, \mathrm{C}_{6} \mathrm{H}_{5}\right), 7.36$ $\left(\mathrm{d}, J=2.0 \mathrm{~Hz}, 1 \mathrm{H}, \mathrm{C}_{6} \mathrm{H}_{3}\right), 7.55\left(\mathrm{dd}, J=2.0,8.4 \mathrm{~Hz}, 1 \mathrm{H}, \mathrm{C}_{6} \mathrm{H}_{3}\right), 7.62\left(\mathrm{dd}, J=1.2,8.0 \mathrm{~Hz}, 2 \mathrm{H}, \mathrm{C}_{6} \mathrm{H}_{5}\right) \mathrm{ppm}$. ${ }^{13} \mathrm{C}\left\{{ }^{1} \mathrm{H}\right\} \operatorname{NMR}\left(\mathrm{C}_{6} \mathrm{D}_{6}\right)$ : $\delta 16.19,19.39,25.23,26.45,33.59,37.80,43.73,51.81,112.53,119.36,126.29$, 
$126.76,128.18,129.02,129.81,130.25,141.92,145.76,177.41,205.30$ ppm. Anal. Calc. $\left(\mathrm{C}_{25} \mathrm{H}_{29} \mathrm{NO}\right)$ : C, 83.52; H, 8.13; N, $3.90 \%$. Found: C, 83.46; H, 7.95; N, $3.69 \%$.

Compound 5d. The compound was synthesized by the same conditions and procedures as for 5a using 4d. It was purified by column chromatography on silica gel eluting with hexane and ethyl acetate (3 : 1). A light yellow solid was obtained (1.07 g, 97 \%). M.p. $47{ }^{\circ} \mathrm{C}$, IR (neat): 3378 (N-H), 1698 $(\mathrm{C}=\mathrm{O}) \mathrm{cm}^{-1} .{ }^{1} \mathrm{H}$ NMR $\left(\mathrm{C}_{6} \mathrm{D}_{6}\right): \delta 0.68\left(\mathrm{~d}, J=7.2 \mathrm{~Hz}, 3 \mathrm{H}, \mathrm{CH}_{3}\right), 1.03-1.17(\mathrm{~m}, 4 \mathrm{H}, \mathrm{Cy}), 1.40-1.46(\mathrm{~m}, 2 \mathrm{H}$, Cy), 1.47 (s, 3H, $\left.\mathrm{CH}_{3}\right), 1.53-1.55$ (m, 2H, Cy), 1.78 (dd, J=2.0, $\left.18.4 \mathrm{~Hz}, 1 \mathrm{H}, \mathrm{CH}_{2}\right), 1.84-1.94$ (m, 2H, Cy), 2.08-2.12 (m, 1H, CH), $2.36\left(\mathrm{dd}, J=7.2,18.4 \mathrm{~Hz}, 1 \mathrm{H}, \mathrm{CH}_{2}\right), 2.97-3.08$ (m, 1H, N-CH), 4.00 (br s, $1 \mathrm{H}, \mathrm{NH}), 6.47\left(\mathrm{~d}, J=8.8 \mathrm{~Hz}, 1 \mathrm{H}, \mathrm{C}_{6} \mathrm{H}_{3}\right), 7.01\left(\mathrm{~d}, J=2.4 \mathrm{~Hz}, 1 \mathrm{H}, \mathrm{C}_{6} \mathrm{H}_{3}\right), 7.21(\mathrm{dd}, J=2.4,8.8 \mathrm{~Hz}, 1 \mathrm{H}$, $\left.\mathrm{C}_{6} \mathrm{H}_{3}\right)$ ppm. ${ }^{13} \mathrm{C}\left\{{ }^{1} \mathrm{H}\right\} \operatorname{NMR}\left(\mathrm{C}_{6} \mathrm{D}_{6}\right): \delta 16.02,19.30,21.15,26.37,33.32,37.80,43.61,51.76,113.08$, 120.46, 120.98, 129.05, 130.94, 138.63, 144.87, 178.02, 204.82 ppm. Anal. Calc. $\left(\mathrm{C}_{19} \mathrm{H}_{24} \mathrm{ClNO}\right)$ : C, 71.80; H, 7.61; N, $4.41 \%$. Found: C, 71.92; H, 7.46; N, $4.34 \%$.

Compound 5e. The compound was synthesized by the same conditions and procedures as for 5a using 4e. It was purified by column chromatography on silica gel eluting with hexane and ethyl acetate (10 : 1). Light yellow oil was obtained (90\%). IR (neat): $3378(\mathrm{~N}-\mathrm{H}), 1698(\mathrm{C}=\mathrm{O}) \mathrm{cm}^{-1} .{ }^{1} \mathrm{H}$ NMR $\left(\mathrm{C}_{6} \mathrm{D}_{6}\right): \delta 0.76\left(\mathrm{~d}, J=7.2 \mathrm{~Hz}, 3 \mathrm{H}, \mathrm{CH}_{3}\right), 1.06-1.20(\mathrm{~m}, 4 \mathrm{H}, \mathrm{Cy}), 1.43-1.48(\mathrm{~m}, 2 \mathrm{H}, \mathrm{Cy}), 1.56(\mathrm{~s}, 3 \mathrm{H}$, $\left.\mathrm{CH}_{3}\right), 1.54-1.62(\mathrm{~m}, 2 \mathrm{H}, \mathrm{Cy}), 1.81$ (dd, $\left.J=2.0,18.4 \mathrm{~Hz}, 1 \mathrm{H}, \mathrm{CH}_{2}\right), 1.86-1.96$ (m, 2H, Cy), 2.18-2.22 (m, $1 \mathrm{H}, \mathrm{CH}), 2.40\left(\mathrm{dd}, J=6.8,18.4 \mathrm{~Hz}, 1 \mathrm{H}, \mathrm{CH}_{2}\right), 2.99-3.08$ (m, 1H, N-CH), 3.78 (br s, 1H, NH), 6.48 (dd, $\left.J=4.8,8.8 \mathrm{~Hz}, 1 \mathrm{H}, \mathrm{C}_{6} \mathrm{H}_{3}\right), 6.77\left(\mathrm{dd}, J=3.2,8.8 \mathrm{~Hz}, 1 \mathrm{H}, \mathrm{C}_{6} \mathrm{H}_{3}\right), 6.91\left(\mathrm{td}, J=3.2,8.8 \mathrm{~Hz}, 1 \mathrm{H}, \mathrm{C}_{6} \mathrm{H}_{3}\right)$ ppm. ${ }^{13} \mathrm{C}\left\{{ }^{1} \mathrm{H}\right\} \operatorname{NMR}\left(\mathrm{C}_{6} \mathrm{D}_{6}\right): \delta 16.06,19.30,25.24,26.45,33.53,37.77,43.63,52.21,112.81\left(\mathrm{~d}, J_{\mathrm{CF}}=\right.$ $\left.7.6 \mathrm{~Hz}, \mathrm{C}_{6} \mathrm{H}_{3}\right), 115.51\left(\mathrm{~d},{ }^{2} J_{\mathrm{CF}}=21.2 \mathrm{~Hz}, \mathrm{C}_{6} \mathrm{H}_{3}\right), 117.84\left(\mathrm{~d},{ }^{2} J_{\mathrm{CF}}=21.2 \mathrm{~Hz}, \mathrm{C}_{6} \mathrm{H}_{3}\right), 120.15\left(\mathrm{~d}, J_{\mathrm{CF}}=7.6\right.$ $\left.\mathrm{Hz}, \mathrm{C}_{6} \mathrm{H}_{3}\right), 142.66,154.01,156.33,177.80,204.84$ ppm. Anal. Calc. $\left(\mathrm{C}_{19} \mathrm{H}_{24} \mathrm{FNO}\right): \mathrm{C}, 75.71 ; \mathrm{H}, 8.03 ; \mathrm{N}$, $4.65 \%$. Found: C, 75.84; H, 8.25; N, $4.48 \%$.

Compound 5f. The compound was synthesized by the same conditions and procedures as for 5a using 4f. It was purified by column chromatography on silica gel eluting with hexane and ethyl acetate (5 : 1). Light yellow oil was obtained (96\%). IR (neat): $3407(\mathrm{~N}-\mathrm{H}), 1697(\mathrm{C}=\mathrm{O}) \mathrm{cm}^{-1} .{ }^{1} \mathrm{H}$ NMR $\left(\mathrm{CDCl}_{3}\right): \delta 1.30\left(\mathrm{~d}, J=6.8 \mathrm{~Hz}, 3 \mathrm{H}, \mathrm{CH}_{3}\right), 1.31\left(\mathrm{~s}, 9 \mathrm{H}, \mathrm{C}\left(\mathrm{CH}_{3}\right)_{3}\right), 2.01\left(\mathrm{~s}, 3 \mathrm{H}, \mathrm{CH}_{3}\right), 2.17$ (dd, $J=2.0$, $18.4 \mathrm{~Hz}, 1 \mathrm{H}, \mathrm{CH}_{2}$ ), 2.80 (dd, $J=6.4,18.4 \mathrm{~Hz}, 1 \mathrm{H}, \mathrm{CH}_{2}$ ), 2.93 (br quintet, $\left.J=6.4 \mathrm{~Hz}, 1 \mathrm{H}, \mathrm{CH}\right), 3.44$ (s, $1 \mathrm{H}, \mathrm{NH}), 6.75\left(\mathrm{td}, J=1.2,7.2 \mathrm{~Hz}, 1 \mathrm{H}, \mathrm{C}_{6} \mathrm{H}_{4}\right), 6.87\left(\mathrm{~d}, J=6.8 \mathrm{~Hz}, 1 \mathrm{H}, \mathrm{C}_{6} \mathrm{H}_{4}\right), 6.98(\mathrm{~d}, J=8.4 \mathrm{~Hz}, 1 \mathrm{H}$, $\left.\mathrm{C}_{6} \mathrm{H}_{4}\right) 7.17\left(\mathrm{td}, J=1.6,7.6 \mathrm{~Hz}, 1 \mathrm{H}, \mathrm{C}_{6} \mathrm{H}_{4}\right) \mathrm{ppm} .{ }^{13} \mathrm{C}\left\{{ }^{1} \mathrm{H}\right\} \mathrm{NMR}\left(\mathrm{CDCl}_{3}\right): \delta 16.16,19.55,29.94,37.50$, 43.52, 51.26, 115.50, 117.20, 119.71, 127.46, 128.34, 130.29, 144.48, 178.44, 206.44 ppm. Anal. Calc. $\left(\mathrm{C}_{17} \mathrm{H}_{23} \mathrm{NO}\right): \mathrm{C}, 79.33$; H, 9.01; N, $5.44 \%$. Found: C, 79.59; H, 9.35; N, $5.16 \%$. 
Compound 5g. The compound was synthesized by the same conditions and procedures as for 5a using 4g. It was purified by column chromatography on silica gel eluting with hexane and ethyl acetate (10 : 1). Light yellow oil was obtained (88\%). IR (neat): $3407(\mathrm{~N}-\mathrm{H}), 1697(\mathrm{C}=\mathrm{O}) \mathrm{cm}^{-1} .{ }^{1} \mathrm{H}$ NMR $\left(\mathrm{CDCl}_{3}\right): \delta 0.91\left(\mathrm{t}, J=7.6 \mathrm{~Hz}, 6 \mathrm{H}, \mathrm{CH}_{3}\right), 1.32\left(\mathrm{~d}, J=7.6 \mathrm{~Hz}, 3 \mathrm{H}, \mathrm{CH}_{3}\right), 1.43-1.61\left(\mathrm{~m}, 4 \mathrm{H}, \mathrm{CH}_{2}\right), 2.02(\mathrm{~s}$, $3 \mathrm{H}, \mathrm{CH}_{3}$ ), 2.19 (dd, $J=18.4,2.0 \mathrm{~Hz}, 1 \mathrm{H}, \mathrm{CH}_{2}$ ), 2.83 (dd, $J=18.4,6.8 \mathrm{~Hz}, 1 \mathrm{H}, \mathrm{CH}_{2}$ ), 2.95 (br quintet, $J$ $=6.8 \mathrm{~Hz}, 1 \mathrm{H}, \mathrm{CH}$ ), 3.25 (quintet, $J=5.6 \mathrm{~Hz}, 1 \mathrm{H}, \mathrm{N}-\mathrm{CH}), 3.34(\mathrm{~s}, 1 \mathrm{H}, \mathrm{NH}), 6.67(\mathrm{~d}, J=7.6 \mathrm{~Hz}, 1 \mathrm{H}$, $\left.\mathrm{C}_{6} \mathrm{H}_{4}\right), 6.68\left(\mathrm{t}, J=7.6 \mathrm{~Hz}, 1 \mathrm{H}, \mathrm{C}_{6} \mathrm{H}_{4}\right), 6.86\left(\mathrm{~d}, J=6.4 \mathrm{~Hz}, 1 \mathrm{H}, \mathrm{C}_{6} \mathrm{H}_{4}\right), 7.12(\mathrm{td}, J=1.2,7.6 \mathrm{~Hz}, 1 \mathrm{H}$, $\left.\mathrm{C}_{6} \mathrm{H}_{4}\right)$ ppm. ${ }^{13} \mathrm{C}\left\{{ }^{1} \mathrm{H}\right\} \mathrm{NMR}\left(\mathrm{CDCl}_{3}\right): \delta 10.09,16.28,19.67,26.42,37.62,43.59,55.05,110.98,115.91$, 127.49, 128.96, 129.51, 130.36, 134.49, 178.76, 206.66 ppm. Anal. Calc. $\left(\mathrm{C}_{18} \mathrm{H}_{25} \mathrm{NO}\right)$ : C, 79.66; H, 9.28; N, $5.16 \%$. Found: C, 79.84; H, 9.42; N, $5.38 \%$.

Compound 6a. Anhydrous $\mathrm{CeCl}_{3}(5.27 \mathrm{~g}, 21.4 \mathrm{mmol})$ and THF $(24 \mathrm{ml})$ were added into a Schlenk flask inside a glovebox. The flask was brought out and the slurry was cooled to $-78^{\circ} \mathrm{C}$. MeLi (13.4 $\mathrm{mL}$, $21.4 \mathrm{mmol}, 1.6 \mathrm{M}$ solution in diethyl ether w/o LiBr) was added with syringe. The mixture was stirred for 1 hour at $-78{ }^{\circ} \mathrm{C}$. Compound 5 a $(2.02 \mathrm{~g}, 7.13 \mathrm{mmol})$ was added as a solid under weak stream of nitrogen gas. After the mixture was stirred for 2 hours at $-78^{\circ} \mathrm{C}$, it was transferred to a separatory funnel containing water $(20 \mathrm{ml})$ and ethyl acetate $(40 \mathrm{~mL})$. The organic phase was collected and the water phase was further extracted with additional ethyl acetate $(10 \mathrm{~mL} \times 2)$. The combined organic phase was shaken vigorously with aqueous $\mathrm{HCl}(2 \mathrm{~N}, 20 \mathrm{~mL})$ for 2 minutes. Aqueous saturated $\mathrm{NaHCO}_{3}(20 \mathrm{~mL})$ was added carefully to neutralize the solution. The collected organic phase was dried with anhydrous $\mathrm{MgSO}_{4}$ and solvent was removed with rotary evaporator to give a residue which was purified by column chromatography on silica gel eluting with hexane and ethyl acetate (v/v, 10:1). Colorless oil was obtained (1.66 g, $83 \%$ ). The cyclopentadiene compound is unstable and slowly decomposes even in a freezer under $\mathrm{N}_{2}$ atmosphere. So, the compound is used for the next reaction as soon as it is prepared. ${ }^{1} \mathrm{H}$ NMR $\left(\mathrm{CDCl}_{3}\right): \delta 1.06-1.20$ (m, 2H, Cy), 1.21-1.30 (m, 1H, Cy), 1.34-1.46 (m, 2H, Cy), 1.68 (d, $J=$ $\left.1.2 \mathrm{~Hz}, 3 \mathrm{H}, \mathrm{CH}_{3}\right), 1.74-1.81$ (m, 3H, Cy), 1.87 (s, 3H, $\left.\mathrm{CH}_{3}\right), 2.01$ (s, 3H, $\left.\mathrm{CH}_{3}\right), 2.03-2.10$ (m, 2H, Cy), $2.94\left(\mathrm{AB}, J=22.8 \mathrm{H}_{\mathrm{Z}}, 1 \mathrm{H}, \mathrm{CH}_{2}\right), 3.01\left(\mathrm{AB}, J=22.8 \mathrm{H}_{\mathrm{Z}}, 1 \mathrm{H}, \mathrm{CH}_{2}\right), 3.29-3.34(\mathrm{~m}, 1 \mathrm{H}, \mathrm{N}-\mathrm{CH}), 3.67$ (br s, $1 \mathrm{H}, \mathrm{NH}), 6.69\left(\mathrm{td}, J=1.2,7.2 \mathrm{~Hz}, 1 \mathrm{H}, \mathrm{C}_{6} \mathrm{H}_{4}\right), 6.71\left(\mathrm{~d}, J=8.0 \mathrm{~Hz}, 1 \mathrm{H}, \mathrm{C}_{6} \mathrm{H}_{4}\right), 6.93(\mathrm{dd}, J=1.6,7.2 \mathrm{~Hz}$, $\left.1 \mathrm{H}, \mathrm{C}_{6} \mathrm{H}_{4}\right), 7.20\left(\mathrm{ddd}, J=1.6,7.2,8.0 \mathrm{~Hz}, 1 \mathrm{H}, \mathrm{C}_{6} \mathrm{H}_{4}\right) \mathrm{ppm} .{ }^{13} \mathrm{C}\left\{{ }^{1} \mathrm{H}\right\} \operatorname{NMR}\left(\mathrm{CDCl}_{3}\right): \delta 11.70,13.73$, $14.47,25.13,25.17,26.05,33.17,33.50,48.81,51.50,110.18,115.50,122.53,127.76,130.01,133.11$, $135.64,136.80,139.66,144.86 \mathrm{ppm}$.

Compound 6b. The compound was synthesized by the same conditions and procedures as for $\mathbf{6 a}$ using 5b. It was purified by column chromatography on silica gel eluting with hexane and ethyl acetate (30 : 1). A light yellow solid was obtained (70 \%). M.p. $62-63{ }^{\circ} \mathrm{C} .{ }^{1} \mathrm{H}$ NMR $\left(\mathrm{C}_{6} \mathrm{D}_{6}\right): \delta 0.92-1.04(\mathrm{~m}, 3 \mathrm{H}$, Cy), 1.12-1.22 (m, 2H, Cy), 1.40-1.48 (m, 1H, Cy), 1.50-1.57 (m, 2H, Cy), 1.81 (s, 3H, $\left.\mathrm{CH}_{3}\right), 1.88$ (s, 
$\left.3 \mathrm{H}, \mathrm{CH}_{3}\right), 1.90\left(\mathrm{~s}, 3 \mathrm{H}, \mathrm{CH}_{3}\right), 1.93-2.01(\mathrm{~m}, 2 \mathrm{H}, \mathrm{Cy}), 2.28\left(\mathrm{~s}, 3 \mathrm{H}, \mathrm{CH}_{3}\right), 2.72\left(\mathrm{AB}, J=22.8 \mathrm{H}_{\mathrm{Z}}, 1 \mathrm{H}, \mathrm{CH}_{2}\right)$, $2.80\left(\mathrm{AB}, J=22.8 \mathrm{H}_{\mathrm{Z}}, 1 \mathrm{H}, \mathrm{CH}_{2}\right), 3.16-3.25(\mathrm{~m}, 1 \mathrm{H}, \mathrm{N}-\mathrm{CH}), 3.65$ (br d, $\left.J=8.0 \mathrm{~Hz}, 1 \mathrm{H}, \mathrm{NH}\right), 6.70(\mathrm{~d}, J$ $\left.=8.0 \mathrm{~Hz}, 1 \mathrm{H}, \mathrm{C}_{6} \mathrm{H}_{3}\right), 6.93\left(\mathrm{~d}, J=2.0 \mathrm{~Hz}, 1 \mathrm{H}, \mathrm{C}_{6} \mathrm{H}_{3}\right), 7.07\left(\mathrm{dd}, J=2.0,8.0 \mathrm{~Hz}, 1 \mathrm{H}, \mathrm{C}_{6} \mathrm{H}_{3}\right) \mathrm{ppm} .{ }^{13} \mathrm{C}\left\{{ }^{1} \mathrm{H}\right\}$ $\operatorname{NMR}\left(\mathrm{C}_{6} \mathrm{D}_{6}\right): \delta 12.19,13.90,14.76,20.95,25.53,26.46,33.97,49.07,51.96,111.28,123.27,124.94$, $129.02,131.14,133.11,136.46,136.62,141.11,143.40 \mathrm{ppm}$.

Compound 6c. The compound was synthesized by the same conditions and procedures as for $\mathbf{6 a}$ using 5c. It was purified by column chromatography on silica gel eluting with hexane and ethyl acetate (10 : 1). A light yellow oil was obtained (75\%). ${ }^{1} \mathrm{H}$ NMR $\left(\mathrm{C}_{6} \mathrm{D}_{6}\right)$ : $\delta$ 0.87-1.04 (m, 3H, Cy), 1.11-1.23 (m, 2H, Cy), 1.41-1.47 (m, 1H, Cy), 1.50-1.58 (m, 2H, Cy), 1.80 (s, 3H, CH $\left.\mathrm{CH}_{3}\right), 1.88$ (s, 3H, $\left.\mathrm{CH}_{3}\right), 1.90$ (s, $\left.3 \mathrm{H}, \mathrm{CH}_{3}\right), 1.93-2.00(\mathrm{~m}, 2 \mathrm{H}, \mathrm{Cy}), 2.72\left(\mathrm{AB}, J=22.8 \mathrm{H}_{\mathrm{Z}}, 1 \mathrm{H}, \mathrm{CH}_{2}\right), 2.80\left(\mathrm{AB}, J=22.8 \mathrm{H}_{\mathrm{Z}}, 1 \mathrm{H}, \mathrm{CH}_{2}\right)$, 3.17-3.28 (m, 1H, N-CH), 3.86 (br d, $J=8.0 \mathrm{~Hz}, 1 \mathrm{H}, \mathrm{NH}), 6.77$ (d, $\left.J=8.0 \mathrm{~Hz}, 1 \mathrm{H}, \mathrm{C}_{6} \mathrm{H}_{3}\right), 7.13(\mathrm{t}, J=$ $\left.8.0 \mathrm{~Hz}, 1 \mathrm{H}, \mathrm{C}_{6} \mathrm{H}_{5}\right), 7.27$ (t, $\left.J=8.0 \mathrm{~Hz}, 2 \mathrm{H}, \mathrm{C}_{6} \mathrm{H}_{5}\right), 7.48$ (d, $\left.J=2.4 \mathrm{~Hz}, 1 \mathrm{H}, \mathrm{C}_{6} \mathrm{H}_{3}\right), 7.59$ (dd, $J=2.4,8.0$ $\left.\mathrm{Hz}, 1 \mathrm{H}, \mathrm{C}_{6} \mathrm{H}_{3}\right), 7.64\left(\mathrm{~d}, J=8.0 \mathrm{~Hz}, 2 \mathrm{H}, \mathrm{C}_{6} \mathrm{H}_{5}\right) \mathrm{ppm} .{ }^{13} \mathrm{C}\left\{{ }^{1} \mathrm{H}\right\} \mathrm{NMR}\left(\mathrm{C}_{6} \mathrm{D}_{6}\right): \delta 12.21,13.90,14.78,25.42$, $25.45,26.35,33.78,33.81,49.13,51.67,111.22$, 123.43, 126.09, 126.60, 127.22, 128.98, 129.23, $129.35,133.45,136.29,137.15,140.73,142.05,145.00 \mathrm{ppm}$.

Compound 6d. The compound was synthesized by the same conditions and procedures as for $\mathbf{6 a}$ using 5d. It was purified by column chromatography on silica gel eluting with hexane and ethyl acetate (10 : 1). A light yellow solid was obtained (60 \%). M.p. $69{ }^{\circ} \mathrm{C} .{ }^{1} \mathrm{H}$ NMR $\left(\mathrm{C}_{6} \mathrm{D}_{6}\right): \delta 0.80-0.90(\mathrm{~m}, 2 \mathrm{H}$, Cy), 0.94-1.01 (m, 1H, Cy), 1.08-1.18 (m, 3H, Cy), 1.40-1.51 (m, 4H, Cy), 1.67 (s, 3H, $\left.\mathrm{CH}_{3}\right), 1.77$ (s, $\left.3 \mathrm{H}, \mathrm{CH}_{3}\right), 1.83\left(\mathrm{~s}, 3 \mathrm{H}, \mathrm{CH}_{3}\right), 2.61\left(\mathrm{AB}, J=22.8 \mathrm{H}_{\mathrm{Z}}, 1 \mathrm{H}, \mathrm{CH}_{2}\right), 2.71\left(\mathrm{AB}, J=22.8 \mathrm{H}_{\mathrm{Z}}, 1 \mathrm{H}, \mathrm{CH}_{2}\right), 2.99-$ 3.07 (m, 1H, N-CH), 3.68 (br d, $J=8.0 \mathrm{~Hz}, 1 \mathrm{H}, \mathrm{NH}), 6.44$ (d, $\left.J=8.8 \mathrm{~Hz}, 1 \mathrm{H}, \mathrm{C}_{6} \mathrm{H}_{3}\right), 7.07$ (d, $J=2.4 \mathrm{~Hz}$, $\left.1 \mathrm{H}, \mathrm{C}_{6} \mathrm{H}_{3}\right), 7.17\left(\mathrm{dd}, J=2.4,8.8 \mathrm{~Hz}, 1 \mathrm{H}, \mathrm{C}_{6} \mathrm{H}_{3}\right) \mathrm{ppm},{ }^{13} \mathrm{C}\left\{{ }^{1} \mathrm{H}\right\} \mathrm{NMR}\left(\mathrm{C}_{6} \mathrm{D}_{6}\right): \delta 11.93,13.82,14.54,25.36$, 25.39 , 26.29, 33.56, 33.59, 49.10, 51.65, 111.77, 120.79, 124.66, 128.24, 130.18, 133.70, 135.67, $137.73,139.52,144.15 \mathrm{ppm}$.

Compound 6e. The compound was synthesized by the same conditions and procedures as for $\mathbf{6 a}$ using 5e. It was purified by column chromatography on silica gel eluting with hexane and ethyl acetate (10 : 1). A yellow oil was obtained (41\%). ${ }^{1} \mathrm{H}$ NMR $\left(\mathrm{C}_{6} \mathrm{D}_{6}\right): \delta$ 0.85-0.92 (m, 2H, Cy), 0.95-1.01 (m, 1H, Cy), 1.08-1.18 (m, 2H, Cy), 1.41-1.46 (m, 1H, Cy), 1.48-1.54 (m, 2H, Cy), 1.71 (d, J=0.4 Hz, 3H, $\left.\mathrm{CH}_{3}\right), 1.80\left(\mathrm{~s}, 3 \mathrm{H}, \mathrm{CH}_{3}\right), 1.82\left(\mathrm{~s}, 3 \mathrm{H}, \mathrm{CH}_{3}\right), 1.84-1.90(\mathrm{~m}, 2 \mathrm{H}, \mathrm{Cy}), 2.62\left(\mathrm{AB}, J=22.8 \mathrm{H}_{\mathrm{Z}}, 1 \mathrm{H}, \mathrm{CH}_{2}\right)$, $2.72\left(\mathrm{AB}, J=22.8 \mathrm{H}_{\mathrm{Z}}, 1 \mathrm{H}, \mathrm{CH}_{2}\right), 3.01-3.09(\mathrm{~m}, 1 \mathrm{H}, \mathrm{N}-\mathrm{CH}), 3.57$ (br d, $\left.J=8.0 \mathrm{~Hz}, 1 \mathrm{H}, \mathrm{NH}\right), 6.45$ (dd, $J$ $\left.=4.8,9.2 \mathrm{~Hz}, 1 \mathrm{H}, \mathrm{C}_{6} \mathrm{H}_{3}\right), 6.88\left(\mathrm{dd}, J=3.2,9.2 \mathrm{~Hz}, 1 \mathrm{H}, \mathrm{C}_{6} \mathrm{H}_{3}\right), 6.94\left(\mathrm{td}, J=3.2,8.4 \mathrm{~Hz}, 1 \mathrm{H}, \mathrm{C}_{6} \mathrm{H}_{3}\right) \mathrm{ppm}$. ${ }^{13} \mathrm{C}\left\{{ }^{1} \mathrm{H}\right\} \operatorname{NMR}\left(\mathrm{C}_{6} \mathrm{D}_{6}\right): \delta 11.96,13.78,14.54,25.40,25.43,26.34,33.75,33.78,49.05,52.14,111.50(\mathrm{~d}$, 
$\left.J_{\mathrm{CF}}=7.6 \mathrm{~Hz}, \mathrm{C}_{6} \mathrm{H}_{3}\right), 114.64\left(\mathrm{~d},{ }^{2} J_{\mathrm{CF}}=21.2 \mathrm{~Hz}, \mathrm{C}_{6} \mathrm{H}_{3}\right), 117.15\left(\mathrm{~d},{ }^{2} J_{\mathrm{CF}}=21.2 \mathrm{~Hz}, \mathrm{C}_{6} \mathrm{H}_{3}\right), 133.59,135.78$, $137.50,139.85,142.02,154.21,156.45 \mathrm{ppm}$.

Compound 6f. The compound was synthesized by the same conditions and procedures as for $\mathbf{6 a}$ using 5f. It was purified by column chromatography on silica gel eluting with hexane and ethyl acetate (20 : 1). A colorless oil was obtained $(0.461 \mathrm{~g}, 58 \%) .{ }^{1} \mathrm{H} \mathrm{NMR}\left(\mathrm{CDCl}_{3}\right): \delta 1.37\left(\mathrm{~s}, 9 \mathrm{H}, \mathrm{C}\left(\mathrm{CH}_{3}\right)_{3}\right), 1.71$ $\left(\mathrm{s}, 3 \mathrm{H}, \mathrm{CH}_{3}\right), 1.90\left(\mathrm{~s}, 3 \mathrm{H}, \mathrm{CH}_{3}\right), 2.03\left(\mathrm{~s}, 3 \mathrm{H}, \mathrm{CH}_{3}\right), 2.96\left(\mathrm{AB}, J=22.8 \mathrm{H}_{\mathrm{Z}}, 1 \mathrm{H}, \mathrm{CH}_{2}\right), 3.03(\mathrm{AB}, J=22.8$ $\mathrm{H}_{\mathrm{Z}}, 1 \mathrm{H}, \mathrm{CH}_{2}$ ), 3.83 (br s, 1H, NH), 6.77 (t, J=7.2 Hz, 1H, $\mathrm{C}_{6} \mathrm{H}_{4}$ ), 6.97 (d, J=7.6 Hz, 1H, $\left.\mathrm{C}_{6} \mathrm{H}_{4}\right), 7.01$ $\left(\mathrm{d}, J=8.0 \mathrm{~Hz}, 1 \mathrm{H}, \mathrm{C}_{6} \mathrm{H}_{4}\right), 7.21\left(\mathrm{t}, J=7.2 \mathrm{~Hz}, 1 \mathrm{H}, \mathrm{C}_{6} \mathrm{H}_{4}\right) \mathrm{ppm} .{ }^{13} \mathrm{C}\left\{{ }^{1} \mathrm{H}\right\} \mathrm{NMR}\left(\mathrm{CDCl}_{3}\right): \delta 11.71,13.70$, 14.46 , 30.09, 48.75, 50.96, 113.93, 116.31, 124.62, 127.24, 129.99, 133.25, 135.44, 136.62, 140.01, $144.70 \mathrm{ppm}$.

Compound 6g. The compound was synthesized by the same conditions and procedures as for $\mathbf{6 a}$ using 5g. It was purified by column chromatography on silica gel eluting with hexane and triethylamine (v/v, 50:1). A colorless oil was obtained (60\%). ${ }^{1} \mathrm{H}$ NMR $\left(\mathrm{CDCl}_{3}\right): \delta 0.91\left(\mathrm{t}, J=7.6 \mathrm{~Hz}, 6 \mathrm{H}, \mathrm{CH}_{3}\right)$, 1.45-1.63 (m, 4H, $\left.\mathrm{CH}_{2}\right), 1.68\left(\mathrm{~s}, 3 \mathrm{H}, \mathrm{CH}_{3}\right), 1.87\left(\mathrm{~s}, 3 \mathrm{H}, \mathrm{CH}_{3}\right), 2.01\left(\mathrm{~s}, 3 \mathrm{H}, \mathrm{CH}_{3}\right), 2.95(\mathrm{AB}, J=22.4 \mathrm{~Hz}$, $1 \mathrm{H}, \mathrm{CH}_{2}$ ), $3.02\left(\mathrm{AB}, J=22.4 \mathrm{~Hz}, 1 \mathrm{H}, \mathrm{CH}_{2}\right.$ ), 3.30 (br quintet, $J=6.0 \mathrm{~Hz}, 1 \mathrm{H}, \mathrm{N}-\mathrm{CH}$ ), 3.56 (br s, 1H, $\mathrm{NH}), 6.67\left(\mathrm{~d}, J=7.6 \mathrm{H}_{\mathrm{Z}}, 1 \mathrm{H}, \mathrm{C}_{6} \mathrm{H}_{4}\right), 6.68\left(\mathrm{t}, J=7.6 \mathrm{~Hz}, 1 \mathrm{H}, \mathrm{C}_{6} \mathrm{H}_{4}\right), 6.92\left(\mathrm{dd}, J=1.6,7.6 \mathrm{~Hz}, 1 \mathrm{H}, \mathrm{C}_{6} \mathrm{H}_{4}\right)$, $7.20\left(\mathrm{td}, J=1.6,7.6 \mathrm{~Hz}, 1 \mathrm{H}, \mathrm{C}_{6} \mathrm{H}_{4}\right) \mathrm{ppm},{ }^{13} \mathrm{C}\left\{{ }^{1} \mathrm{H}\right\} \mathrm{NMR}\left(\mathrm{CDCl}_{3}\right): \delta 10.15,10.23,13.69,14.36,26.73$, $26.83,48.78,54.95,109.66,115.10,122.42,127.75,129.88,132.94,135.75,136.97,139.76,145.66$ ppm.

Complex 7a. Compound 6a $(0.196 \mathrm{~g}, 0.696 \mathrm{mmol})$ and $\mathrm{Ti}\left(\mathrm{NMe}_{2}\right)_{4}(0.156 \mathrm{~g}, 0.696 \mathrm{mmol})$ were dissolved in toluene $(2 \mathrm{~mL})$ and the flask was sealed. The solution was heated for 2 days at $80{ }^{\circ} \mathrm{C}$. Removal of solvent gave red solid. The NMR data for the intermediate bis(dimethylamido)titanium complex: ${ }^{1} \mathrm{H}$ NMR $\left(\mathrm{C}_{6} \mathrm{D}_{6}\right)$ : $\delta$ 0.86-1.01 (m, 1H, Cy), 1.10-1.25 (m, 2H, Cy), 1.34-1.49 (m, 3H, Cy), 1.64-1.74 (m, 2H, Cy), 1.77 (s, 3H, CH 3 ), 1.86 (s, 3H, $\mathrm{CH}_{3}$ ), 1.95 (s, 3H, $\left.\mathrm{CH}_{3}\right), 1.99-2.09$ (m, 2H, Cy), 2.89 (s, 6H, $\mathrm{NCH}_{3}$ ), 3.07 (br s, 6H, $\mathrm{NCH}_{3}$ ), 5.65 (br s, 1H, Cp-H), 6.68-6.84 (br s, 1H, $\mathrm{C}_{6} \mathrm{H}_{4}$ ), 6.86 (td, $J$ $\left.=0.8,7.2 \mathrm{~Hz}, 1 \mathrm{H}, \mathrm{C}_{6} \mathrm{H}_{4}\right), 7.18\left(\mathrm{dd}, J=1.6,7.2 \mathrm{~Hz}, 1 \mathrm{H}, \mathrm{C}_{6} \mathrm{H}_{4}\right), 7.24\left(\mathrm{td}, J=1.6,7.2 \mathrm{~Hz}, 1 \mathrm{H}, \mathrm{C}_{6} \mathrm{H}_{4}\right) \mathrm{ppm}$. To the flask containing the bis(dimethylamido)titanium complex was added toluene $(2 \mathrm{~mL})$ and $\mathrm{Me}_{2} \mathrm{SiCl}_{2}$ (0.269 g, $\left.2.09 \mathrm{mmol}\right)$ successively at room temperature. After the solution was stirred for 4 hours at room temperature, solvent was removed by vacuum. Analytically pure red crystals were isolated by recrystallization in hexane at $-30{ }^{\circ} \mathrm{C}(0.183 \mathrm{~g}$, overall yield, $66 \%)$. ${ }^{1} \mathrm{H}$ NMR $\left(\mathrm{C}_{6} \mathrm{D}_{6}\right): \delta 0.83-$ $1.00(\mathrm{~m}, 2 \mathrm{H}, \mathrm{Cy}), 1.35-1.51$ (m, 3H, Cy), 1.64 (s, 3H, $\left.\mathrm{CH}_{3}\right), 1.66-1.74(\mathrm{~m}, 3 \mathrm{H}, \mathrm{Cy}), 1.75\left(\mathrm{~s}, 3 \mathrm{H}, \mathrm{CH}_{3}\right)$, 1.81-1.95 (m, 2H, Cy), 2.09 (s, 3H, CH $), 5.46-5.58(\mathrm{~m}, 1 \mathrm{H}, \mathrm{N}-\mathrm{CH}), 6.06$ (s, 1H, Cp-H), 6.65 (d, J = 7.2 $\left.\mathrm{Hz}, 1 \mathrm{H}, \mathrm{C}_{6} \mathrm{H}_{4}\right), 6.95\left(\mathrm{td}, J=0.8,7.2 \mathrm{~Hz}, 1 \mathrm{H}, \mathrm{C}_{6} \mathrm{H}_{4}\right), 7.07\left(\mathrm{dd}, J=2.0,7.2 \mathrm{~Hz}, 1 \mathrm{H}, \mathrm{C}_{6} \mathrm{H}_{4}\right), 7.11(\mathrm{td}, J=$ 
2.0, 7.2 Hz, 1H, $\left.\mathrm{C}_{6} \mathrm{H}_{4}\right)$ ppm. ${ }^{13} \mathrm{C}\left\{{ }^{1} \mathrm{H}\right\}$ NMR $\left(\mathrm{C}_{6} \mathrm{D}_{6}\right): \delta$ 12.38, 14.48, 14.82, 25.81, 27.08, 27.51, 59.57, $111.11,118.50,123.05,128.49,128.99,131.56,132.17,142.13,142.93,143.42$, 164.02 ppm. Anal. Calc. $\left(\mathrm{C}_{20} \mathrm{H}_{25} \mathrm{Cl}_{2} \mathrm{NTi}\right)$ : C, 60.33; H, 6.33; N, $3.52 \%$. Found: C, 60.19; H, 6.52; N, $3.29 \%$.

Complex 7b. The compound was synthesized by the same conditions and procedures as for $7 \mathbf{a}$ using 6b. It was purified by recrystallization in hexane at $-30{ }^{\circ} \mathrm{C}$. Red crystals were isolated in $59 \%$. The ${ }^{1} \mathrm{H}$ NMR datum for the intermediate bis(dimethylamido)titanium complex: ${ }^{1} \mathrm{H} N M R\left(\mathrm{C}_{6} \mathrm{D}_{6}\right)$ : $\delta$ 0.88-1.01 (m, 1H, Cy), 1.15-1.26 (m, 2H, Cy), 1.36-1.50 (m, 3H, Cy), 1.65-1.75 (m, 2H, Cy), 1.81 (s, 3H, $\left.\mathrm{CH}_{3}\right), 1.82-$ $1.88(\mathrm{~m}, 2 \mathrm{H}, \mathrm{Cy}), 1.89$ (s, 3H, $\left.\mathrm{CH}_{3}\right), 1.99\left(\mathrm{~s}, 3 \mathrm{H}, \mathrm{CH}_{3}\right), 2.32\left(\mathrm{~s}, 3 \mathrm{H}, \mathrm{CH}_{3}\right), 2.92\left(\mathrm{~s}, 6 \mathrm{H}, \mathrm{NCH}_{3}\right), 3.04$ (br s, $6 \mathrm{H}, \mathrm{NCH}_{3}$ ), 5.67 (br s, 1H, Cp-H), 6.64-6.76 (br s, 1H, $\left.\mathrm{C}_{6} \mathrm{H}_{3}\right), 7.02$ (s, 1H, $\left.\mathrm{C}_{6} \mathrm{H}_{3}\right), 7.11$ (d, J = 7.6 Hz, $\left.1 \mathrm{H}, \mathrm{C}_{6} \mathrm{H}_{3}\right) \mathrm{ppm}$. The analytical data for $7 \mathbf{b}:{ }^{1} \mathrm{H}$ NMR $\left(\mathrm{C}_{6} \mathrm{D}_{6}\right): \delta 0.84-1.00(\mathrm{~m}, 2 \mathrm{H}, \mathrm{Cy}), 1.37-1.53(\mathrm{~m}, 3 \mathrm{H}$, Cy), 1.69 (s, 3H, $\left.\mathrm{CH}_{3}\right), 1.71-1.76$ (m, 2H, Cy), 1.80 (s, 3H, $\left.\mathrm{CH}_{3}\right), 1.85-1.97$ (m, 2H, Cy), 2.10-2.18 (m, $1 \mathrm{H}, \mathrm{Cy}), 2.11\left(\mathrm{~s}, 3 \mathrm{H}, \mathrm{CH}_{3}\right), 2.21\left(\mathrm{~s}, 3 \mathrm{H}, \mathrm{CH}_{3}\right), 5.57$ (m, 1H, N-CH), 6.09 (s, 1H, Cp-H), 6.60 (d, J = 8.4 $\left.\mathrm{Hz}, 1 \mathrm{H}, \mathrm{C}_{6} \mathrm{H}_{3}\right), 6.91\left(\mathrm{~s}, 1 \mathrm{H}, \mathrm{C}_{6} \mathrm{H}_{3}\right), 6.94\left(\mathrm{~d}, J=8.4 \mathrm{~Hz}, 1 \mathrm{H}, \mathrm{C}_{6} \mathrm{H}_{3}\right) \mathrm{ppm} .{ }^{13} \mathrm{C}\left\{{ }^{1} \mathrm{H}\right\} \mathrm{NMR}\left(\mathrm{C}_{6} \mathrm{D}_{6}\right): \delta 12.45$, $14.50,14.89,20.69,25.85,27.10,27.58,59.59,110.77,118.38,128.87,129.68,131.37,132.55,132.75$, 142.06, 142.64, 143.11, 161.82 ppm. Anal. Calc. $\left(\mathrm{C}_{21} \mathrm{H}_{27} \mathrm{Cl}_{2} \mathrm{NTi}\right)$ : C, 61.19; H, 6.60; N, 3.40 \%. Found: C, 60.94; H, 6.54; N, $3.61 \%$.

Complex 7c. The compound was synthesized by the same conditions and procedures as for 7a using 6c. It was purified by trituration in pentane. Red powder was isolated in $87 \%$ yield. The ${ }^{1} \mathrm{H}$ NMR datum for the intermediate bis(dimethylamido)titanium complex: ${ }^{1} \mathrm{H}$ NMR $\left(\mathrm{C}_{6} \mathrm{D}_{6}\right): \delta 0.84-0.98(\mathrm{~m}, 1 \mathrm{H}$, Cy), 1.14-1.29 (m, 2H, Cy), 1.37-1.52 (m, 3H, Cy), 1.68-1.77 (m, 2H, Cy), 1.79 (s, 3H, $\left.\mathrm{CH}_{3}\right), 1.89$ (s, $\left.3 \mathrm{H}, \mathrm{CH}_{3}\right), 1.96\left(\mathrm{~s}, 3 \mathrm{H}, \mathrm{CH}_{3}\right), 2.04-2.16(\mathrm{~m}, 2 \mathrm{H}, \mathrm{Cy}), 2.91\left(\mathrm{~s}, 6 \mathrm{H}, \mathrm{NCH}_{3}\right), 3.13\left(\mathrm{~s}, 6 \mathrm{H}, \mathrm{NCH}_{3}\right), 5.68(\mathrm{~s}$, $1 \mathrm{H}, \mathrm{Cp}-\mathrm{H}$ ), 6.84 (br s, 1H, $\mathrm{C}_{6} \mathrm{H}_{3}$ ), 7.13 (t, J=8.0 Hz, 1H, $\mathrm{C}_{6} \mathrm{H}_{5}$ ), 7.29 (t, J=8.0 Hz, 2H, $\mathrm{C}_{6} \mathrm{H}_{5}$ ), 7.51 (d, $\left.J=2.0 \mathrm{~Hz}, 1 \mathrm{H}, \mathrm{C}_{6} \mathrm{H}_{3}\right), 7.58\left(\mathrm{dd}, J=2.0,8.0 \mathrm{~Hz}, 1 \mathrm{H}, \mathrm{C}_{6} \mathrm{H}_{3}\right), 7.69\left(\mathrm{dd}, J=2.0,8.0 \mathrm{~Hz}, 2 \mathrm{H}, \mathrm{C}_{6} \mathrm{H}_{5}\right) \mathrm{ppm}$. The analytical data for 7c: ${ }^{1} \mathrm{H}$ NMR $\left(\mathrm{C}_{6} \mathrm{D}_{6}\right): \delta$ 0.93-1.06 (m, 2H, Cy), 1.38-1.56 (m, 4H, Cy), $1.70(\mathrm{~s}, 3 \mathrm{H}$, $\left.\mathrm{CH}_{3}\right), 1.72-1.80(\mathrm{~m}, 2 \mathrm{H}, \mathrm{Cy}), 1.81\left(\mathrm{~s}, 3 \mathrm{H}, \mathrm{CH}_{3}\right), 1.88-2.03(\mathrm{~m}, 2 \mathrm{H}, \mathrm{Cy}), 2.13\left(\mathrm{~s}, 3 \mathrm{H}, \mathrm{CH}_{3}\right), 5.54(\mathrm{~m}, 1 \mathrm{H}$, $\mathrm{N}-\mathrm{CH}), 6.10(\mathrm{~s}, 1 \mathrm{H}, \mathrm{Cp}-\mathrm{H}), 6.71\left(\mathrm{~d}, J=8.0 \mathrm{~Hz}, 1 \mathrm{H}, \mathrm{C}_{6} \mathrm{H}_{3}\right), 7.20\left(\mathrm{~d}, J=8.0 \mathrm{~Hz}, 1 \mathrm{H}, \mathrm{C}_{6} \mathrm{H}_{5}\right), 7.31(\mathrm{t}, J=$ $\left.8.0 \mathrm{~Hz}, 2 \mathrm{H}, \mathrm{C}_{6} \mathrm{H}_{5}\right), 7.38\left(\mathrm{~d}, J=2.0 \mathrm{~Hz}, 1 \mathrm{H}, \mathrm{C}_{6} \mathrm{H}_{3}\right), 7.44\left(\mathrm{dd}, J=2.0,8.0 \mathrm{~Hz}, 1 \mathrm{H}, \mathrm{C}_{6} \mathrm{H}_{3}\right), 7.58(\mathrm{dd}, J=2.0$, $\left.8.0 \mathrm{~Hz}, 2 \mathrm{H}, \mathrm{C}_{6} \mathrm{H}_{5}\right) \mathrm{ppm} .{ }^{13} \mathrm{C}\left\{{ }^{1} \mathrm{H}\right\}$ NMR $\left(\mathrm{C}_{6} \mathrm{D}_{6}\right): \delta 12.58,14.63,15.03,25.95,27.17,27.68,59.73$, 111.22 , 118.40, 126.81, 126.99, 127.27, 128.79, 129.05, 131.50, 132.68, 136.14, 140.46, 141.77, 142.72, 143.20, 163.14 ppm. Anal. Calc. $\left(\mathrm{C}_{26} \mathrm{H}_{29} \mathrm{Cl}_{2} \mathrm{NTi}\right): \mathrm{C}, 65.84 ; \mathrm{H}, 6.16 ; \mathrm{N}, 2.95 \%$. Found: $\mathrm{C}, 65.92 ; \mathrm{H}$, $6.05 ; \mathrm{N}, 3.13 \%$.

Complex 7d. The compound was synthesized by the same conditions and procedures as for 7a using 6d. It was purified by recrystallization in hexane at $-30{ }^{\circ} \mathrm{C}$. Red crystals were isolated in $73 \%$ yield. 
The ${ }^{1} \mathrm{H}$ NMR datum for the intermediate bis(dimethylamido)titanium complex: ${ }^{1} \mathrm{H} N M R\left(\mathrm{C}_{6} \mathrm{D}_{6}\right): \delta 0.84-$ 0.95 (m, 1H, Cy), 1.13-1.22 (m, 2H, Cy), 1.32-1.43 (m, 3H, Cy), 1.67 (s, 3H, $\left.\mathrm{CH}_{3}\right), 1.77$ (s, 3H, $\left.\mathrm{CH}_{3}\right)$, 1.79-1.84 (m, 2H, Cy), 1.92-2.03 (m, 2H, Cy), 1.90 (s, 3H, $\left.\mathrm{CH}_{3}\right), 2.84$ (s, 6H, $\left.\mathrm{NCH}_{3}\right), 3.03$ (br s, 6H, $\left.\mathrm{NCH}_{3}\right), 5.60(\mathrm{~s}, 1 \mathrm{H}, \mathrm{Cp}-\mathrm{H}), 6.52\left(\right.$ br s, $\left.1 \mathrm{H}, \mathrm{C}_{6} \mathrm{H}_{3}\right), 7.16$ (d, J = 2.4 Hz, 1H, $\left.\mathrm{C}_{6} \mathrm{H}_{3}\right), 7.20$ (dd, J = 2.4, 8.8 $\left.\mathrm{Hz}, 1 \mathrm{H}, \mathrm{C}_{6} \mathrm{H}_{3}\right) \mathrm{ppm}$. The analytical data for $7 \mathrm{~d}:{ }^{1} \mathrm{H}$ NMR $\left(\mathrm{C}_{6} \mathrm{D}_{6}\right): \delta 0.82-0.96(\mathrm{~m}, 2 \mathrm{H}, \mathrm{Cy}), 1.31-1.50(\mathrm{~m}$, 4H, Cy), 1.56 (s, 3H, $\mathrm{CH}_{3}$ ), 1.67 (s, 3H, $\left.\mathrm{CH}_{3}\right), 1.68-1.78$ (m, 2H, Cy), 1.91-2.03 (m, 2H, Cy), 2.04 (s, $\left.3 \mathrm{H}, \mathrm{CH}_{3}\right), 5.39(\mathrm{~m}, 1 \mathrm{H}, \mathrm{N}-\mathrm{CH}), 6.00(\mathrm{~s}, 1 \mathrm{H}, \mathrm{Cp}-\mathrm{H}), 6.40\left(\mathrm{~d}, J=8.8 \mathrm{~Hz}, 1 \mathrm{H}, \mathrm{C}_{6} \mathrm{H}_{3}\right), 7.04$ (d, J = 2.8 Hz, $\left.1 \mathrm{H}, \mathrm{C}_{6} \mathrm{H}_{3}\right), 7.10\left(\mathrm{dd}, J=2.8,8.8 \mathrm{~Hz}, 1 \mathrm{H}, \mathrm{C}_{6} \mathrm{H}_{3}\right) \mathrm{ppm} .{ }^{13} \mathrm{C}\left\{{ }^{1} \mathrm{H}\right\}$ NMR $\left(\mathrm{C}_{6} \mathrm{D}_{6}\right): \delta 12.30,14.44,14.74$, 25.72, 26.97, 27.28, 59.67, 111.71, 118.64, 128.33, 128.45, 129.05, 131.85, 133.38, 140.29, 142.78, 143.28, 162.54 ppm. Anal. Calc. $\left(\mathrm{C}_{20} \mathrm{H}_{24} \mathrm{Cl}_{3} \mathrm{NTi}\right): \mathrm{C}, 55.52 ; \mathrm{H}, 5.59 ; \mathrm{N}, 3.24$ \%. Found: C, 55.38; H, $5.79 ; \mathrm{N}, 3.34 \%$.

Complex 7e. The compound was synthesized by the same conditions and procedures as for $\mathbf{7 a}$ using 6e. It is oily and could not be purified by recrystallization. Extraction with pentane gave clean compound $(90 \%)$. The ${ }^{1} \mathrm{H}$ NMR datum for the intermediate bis(dimethylamido)titanium complex: ${ }^{1} \mathrm{H}$ $\operatorname{NMR}\left(\mathrm{C}_{6} \mathrm{D}_{6}\right): \delta$ 0.82-0.96 (m, 1H, Cy), 1.08-1.23 (m, 2H, Cy), 1.32-1.45 (m, 3H, Cy), $1.70\left(\mathrm{~s}, 3 \mathrm{H}, \mathrm{CH}_{3}\right)$, 1.79 (s, 3H, $\left.\mathrm{CH}_{3}\right), 1.82-1.88$ (m, 2H, Cy), $1.92\left(\mathrm{~s}, 3 \mathrm{H}, \mathrm{CH}_{3}\right), 1.97-2.08$ (m, 2H, Cy), $2.86\left(\mathrm{~s}, 6 \mathrm{H}, \mathrm{NCH}_{3}\right)$, 3.03 (s, 6H, $\mathrm{NCH}_{3}$ ), 5.60 (s, 1H, Cp-H), 6.49 (br s, $\left.1 \mathrm{H}, \mathrm{C}_{6} \mathrm{H}_{3}\right), 6.89-6.95$ (m, 2H, $\left.\mathrm{C}_{6} \mathrm{H}_{3}\right)$ ppm. The analytical data for 7e: ${ }^{1} \mathrm{H}$ NMR $\left(\mathrm{C}_{6} \mathrm{D}_{6}\right): \delta$ 0.88-1.03 (m, 2H, Cy), 1.32-1.47 (m, 2H, Cy), 1.47-1.56 (m, $1 \mathrm{H}, \mathrm{Cy}), 1.61$ (s, 3H, $\left.\mathrm{CH}_{3}\right), 1.71$ (s, 3H, $\left.\mathrm{CH}_{3}\right), 1.72-1.84$ (m, 3H, Cy), 1.93-2.05 (m, 2H, Cy), 2.09 (s, $\left.3 \mathrm{H}, \mathrm{CH}_{3}\right), 5.38-5.47(\mathrm{~m}, 1 \mathrm{H}, \mathrm{N}-\mathrm{CH}), 6.05(\mathrm{~s}, 1 \mathrm{H}, \mathrm{Cp}-\mathrm{H}), 6.43\left(\mathrm{dd}, J=4.8,8.8 \mathrm{~Hz}, 1 \mathrm{H}, \mathrm{C}_{6} \mathrm{H}_{3}\right), 6.79-6.85$ (m, 2H, $\left.\mathrm{C}_{6} \mathrm{H}_{3}\right)$ ppm. ${ }^{13} \mathrm{C}\left\{{ }^{1} \mathrm{H}\right\}$ NMR $\left(\mathrm{C}_{6} \mathrm{D}_{6}\right): \delta 12.61,14.75,15.04,26.03,27.29,27.58,27.66,59.74$, $111.38\left(\mathrm{~d},{ }^{3} J_{\mathrm{CF}}=8.3 \mathrm{~Hz}, \mathrm{C}_{6} \mathrm{H}_{3}\right), 114.80\left(\mathrm{~d},{ }^{2} J_{\mathrm{CF}}=22.8 \mathrm{~Hz}, \mathrm{C}_{6} \mathrm{H}_{3}\right), 116.49\left(\mathrm{~d},{ }^{2} J_{\mathrm{CF}}=23.5 \mathrm{~Hz}, \mathrm{C}_{6} \mathrm{H}_{3}\right)$, $118.62,131.69,133.42\left(\mathrm{~d},{ }^{3} J_{\mathrm{CF}}=8.3 \mathrm{~Hz}, \mathrm{C}_{6} \mathrm{H}_{3}\right), 140.02,142.53,143.00,159.74\left(\mathrm{~d},{ }^{1} J_{\mathrm{CF}}=240.4 \mathrm{~Hz}\right.$, $\left.\mathrm{C}_{6} \mathrm{H}_{3}\right), 160.02 \mathrm{ppm} .{ }^{19} \mathrm{~F}$ NMR $\left(\mathrm{C}_{6} \mathrm{D}_{6}\right): \delta-28.83(\mathrm{dd}, J=7.8,12.4 \mathrm{~Hz}) \mathrm{ppm}$.

Complex 7f. The compound was synthesized by the same conditions and procedures as for 7a using 6f. The reaction between $\operatorname{Ti}\left(\mathrm{NMe}_{2}\right)_{4}$ and $\mathbf{6 f}$ was so slow that complete conversion to the intermediate bis(dimethylamido)titanium complex was achieved by heating for 10 days at $80{ }^{\circ} \mathrm{C}$. Overall yield was $56 \%$. The ${ }^{1} \mathrm{H}$ NMR datum for the intermediate bis(dimethylamido)titanium complex: ${ }^{1} \mathrm{H}$ NMR $\left(\mathrm{C}_{6} \mathrm{D}_{6}\right)$ : $\delta 1.56\left(\mathrm{~s}, 9 \mathrm{H}, \mathrm{C}\left(\mathrm{CH}_{3}\right)_{3}\right), 1.71\left(\mathrm{~s}, 3 \mathrm{H}, \mathrm{CH}_{3}\right), 1.87\left(\mathrm{~s}, 3 \mathrm{H}, \mathrm{CH}_{3}\right), 1.98\left(\mathrm{~s}, 3 \mathrm{H}, \mathrm{CH}_{3}\right), 2.82\left(\mathrm{~s}, 6 \mathrm{H}, \mathrm{NCH}_{3}\right)$, $3.00\left(\mathrm{~s}, 6 \mathrm{H}, \mathrm{NCH}_{3}\right), 5.68(\mathrm{~s}, 1 \mathrm{H}, \mathrm{Cp}-\mathrm{H}), 6.84$ (t, $\left.J=8.0 \mathrm{~Hz}, 1 \mathrm{H}, \mathrm{C}_{6} \mathrm{H}_{4}\right), 6.93\left(\mathrm{~d}, J=8.0 \mathrm{~Hz}, 1 \mathrm{H}, \mathrm{C}_{6} \mathrm{H}_{4}\right)$, $7.15\left(\mathrm{dd}, J=2.0,8.0 \mathrm{~Hz}, 1 \mathrm{H}, \mathrm{C}_{6} \mathrm{H}_{4}\right), 7.22\left(\mathrm{td}, J=2.0,8.0 \mathrm{~Hz}, 1 \mathrm{H}, \mathrm{C}_{6} \mathrm{H}_{4}\right) \mathrm{ppm}$. The analytical data for 7f: ${ }^{1} \mathrm{H}$ NMR $\left(\mathrm{C}_{6} \mathrm{D}_{6}\right): \delta 1.68\left(\mathrm{~s}, 3 \mathrm{H}, \mathrm{CH}_{3}\right), 1.80\left(\mathrm{~s}, 12 \mathrm{H}, \mathrm{C}\left(\mathrm{CH}_{3}\right)_{3}\right.$ and $\left.\mathrm{CH}_{3}\right), 2.12\left(\mathrm{~s}, 3 \mathrm{H}, \mathrm{CH}_{3}\right), 6.21(\mathrm{~s}, 1 \mathrm{H}$, Cp-H), $6.71\left(\mathrm{~d}, J=8.4 \mathrm{~Hz}, 1 \mathrm{H}, \mathrm{C}_{6} \mathrm{H}_{4}\right), 6.93\left(\mathrm{~d}, J=8.4 \mathrm{~Hz}, 1 \mathrm{H}, \mathrm{C}_{6} \mathrm{H}_{4}\right), 6.95\left(\mathrm{t}, J=8.4 \mathrm{~Hz}, 1 \mathrm{H}, \mathrm{C}_{6} \mathrm{H}_{4}\right)$, 
$7.10\left(\mathrm{t}, J=8.4 \mathrm{~Hz}, 1 \mathrm{H}, \mathrm{C}_{6} \mathrm{H}_{4}\right) \mathrm{ppm} .{ }^{13} \mathrm{C}\left\{{ }^{1} \mathrm{H}\right\}$ NMR $\left(\mathrm{C}_{6} \mathrm{D}_{6}\right): \delta 12.65,14.98,15.04,29.98,60.64,115.18$, $122.08,122.46,128.14,128.58,129.89$, 134.47, 141.17, 143.41, 143.91, 163.84 ppm. Anal. Calc. $\left(\mathrm{C}_{18} \mathrm{H}_{23} \mathrm{Cl}_{2} \mathrm{NTi}\right)$ : C, 58.09; H, 6.23; N, $3.76 \%$. Found: C, 58.24; H, 6.54; N, $3.51 \%$.

Complex 7g. The compound was synthesized by the same conditions and procedures as for $\mathbf{7 a}$ using 6g. Analytically pure crystals were isolated in $67 \%$ yield by recrystallization in pentane at $-30{ }^{\circ} \mathrm{C}$. The ${ }^{1} \mathrm{H}$ NMR datum for the intermediate bis(dimethylamido)titanium complex: ${ }^{1} \mathrm{H} N M R\left(\mathrm{C}_{6} \mathrm{D}_{6}\right): \delta 0.89$ (br t, $J=6.8 \mathrm{~Hz}, 3 \mathrm{H}$, pentyl-CH ), 1.07 (br t, $J=6.8 \mathrm{~Hz}, 3 \mathrm{H}$, pentyl- $\left.\mathrm{CH}_{3}\right), 1.74\left(\mathrm{~s}, 3 \mathrm{H}, \mathrm{CH}_{3}\right), 1.76-2.01(\mathrm{~m}, 4 \mathrm{H}$, pentyl- $\left.\mathrm{CH}_{2}\right), 1.87\left(\mathrm{~s}, 3 \mathrm{H}, \mathrm{CH}_{3}\right), 1.91\left(\mathrm{~s}, 3 \mathrm{H}, \mathrm{CH}_{3}\right), 2.89\left(\mathrm{~s}, 6 \mathrm{H}, \mathrm{NCH}_{3}\right), 3.16$ (br s, $\left.6 \mathrm{H}, \mathrm{NCH}_{3}\right), 4.20$ (br s, $1 \mathrm{H}$, pentyl-CH), $5.75(\mathrm{~s}, 1 \mathrm{H}, \mathrm{Cp}-\mathrm{H}), 6.65\left(\mathrm{~d}, J=8.0 \mathrm{~Hz}, 1 \mathrm{H}, \mathrm{C}_{6} \mathrm{H}_{4}\right), 6.86\left(\mathrm{td}, J=1.2,8.0 \mathrm{~Hz}, 1 \mathrm{H}, \mathrm{C}_{6} \mathrm{H}_{4}\right)$, $7.18\left(\mathrm{~d}, J=8.0 \mathrm{~Hz}, 1 \mathrm{H}, \mathrm{C}_{6} \mathrm{H}_{4}\right), 7.19\left(\mathrm{t}, J=8.0 \mathrm{~Hz}, 1 \mathrm{H}, \mathrm{C}_{6} \mathrm{H}_{4}\right) \mathrm{ppm}$. The analytical data for $7 \mathrm{~g}:{ }^{1} \mathrm{H} \mathrm{NMR}$ $\left(\mathrm{C}_{6} \mathrm{D}_{6}\right): \delta 1.01\left(\mathrm{t}, J=7.6 \mathrm{~Hz}, 6 \mathrm{H}\right.$, pentyl- $\left.\mathrm{CH}_{3}\right), 1.66\left(\mathrm{~s}, 3 \mathrm{H}, \mathrm{CH}_{3}\right), 1.78\left(\mathrm{~s}, 3 \mathrm{H}, \mathrm{CH}_{3}\right), 1.79-1.93(\mathrm{~m}, 4 \mathrm{H}$, pentyl- $\left.\mathrm{CH}_{2}\right), 2.10\left(\mathrm{~s}, 3 \mathrm{H}, \mathrm{CH}_{3}\right), 5.43-5.50(\mathrm{~m}, 1 \mathrm{H}$, pentyl-CH), $6.07(\mathrm{~s}, 1 \mathrm{H}, \mathrm{Cp}-\mathrm{H}), 6.43(\mathrm{~d}, J=8.0 \mathrm{~Hz}$, $\left.1 \mathrm{H}, \mathrm{C}_{6} \mathrm{H}_{4}-\mathrm{CH}\right), 6.95\left(\mathrm{t}, J=8.0 \mathrm{~Hz}, 1 \mathrm{H}, \mathrm{C}_{6} \mathrm{H}_{4}-\mathrm{CH}\right), 7.07\left(\mathrm{~d}, J=8.0 \mathrm{~Hz}, 1 \mathrm{H}, \mathrm{C}_{6} \mathrm{H}_{4}\right), 7.08(\mathrm{t}, J=8.0 \mathrm{~Hz}$, $\left.1 \mathrm{H}, \mathrm{C}_{6} \mathrm{H}_{4}\right)$ ppm. ${ }^{13} \mathrm{C}\left\{{ }^{1} \mathrm{H}\right\}$ NMR $\left(\mathrm{C}_{6} \mathrm{D}_{6}\right): \delta 12.45,12.63,14.74,14.90,26.58,62.63,111.27,118.62$, $122.99,128.47,128.74,131.36,131.73,142.16,142.76,143.32,163.92$ ppm. Anal. Calc. $\left(\mathrm{C}_{19} \mathrm{H}_{25} \mathrm{Cl}_{2} \mathrm{NTi}\right): \mathrm{C}, 59.09 ; \mathrm{H}, 6.53$; N, 3.63 \%. Found: C, 59.16; H, 6.76; N, $3.49 \%$.

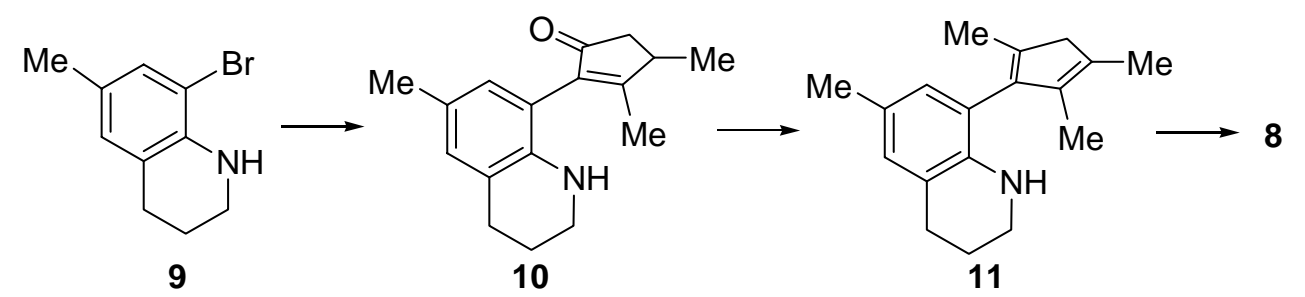

Compound 9. 6-Methyl-1,2,3,4-tetrahydroquinoline (1.16 g, $7.90 \mathrm{mmol})$ was dissolved in $\mathrm{CCl}_{4}$ (4 $\mathrm{mL})$ and the solution was cooled to $-20{ }^{\circ} \mathrm{C}$. N-Bromosuccinimide $(1.41 \mathrm{~g}, 7.90 \mathrm{mmol})$ was added slowly as a solid. ${ }^{5}$ The solution was warmed to room temperature and stirred for 5 hours. The crude mixture was loaded on silica pad and the column chromatography was conducted eluting with methylene chloride and hexane (1: 1). Light yellow oil was obtained (0.710 g, $40 \%)$. IR (neat): $3413(\mathrm{~N}-\mathrm{H}) \mathrm{cm}^{-1}$. ${ }^{1} \mathrm{H}$ NMR $\left(\mathrm{C}_{6} \mathrm{D}_{6}\right): \delta 1.42-1.52\left(\mathrm{~m}, 2 \mathrm{H}, \mathrm{CH}_{2}\right), 2.00\left(\mathrm{~s}, 3 \mathrm{H}, \mathrm{CH}_{3}\right), 2.39\left(\mathrm{t}, J=6.4 \mathrm{~Hz}, 2 \mathrm{H}, \mathrm{CH}_{2}\right), 2.75(\mathrm{dt}, J$ $\left.=2.8,8.4 \mathrm{~Hz}, 2 \mathrm{H}, \mathrm{N}-\mathrm{CH}_{2}\right), 4.04($ br s, $1 \mathrm{H}, \mathrm{NH}), 6.51\left(\mathrm{~s}, 1 \mathrm{H}, \mathrm{C}_{6} \mathrm{H}_{2}\right), 7.09\left(\mathrm{~s}, 1 \mathrm{H}, \mathrm{C}_{6} \mathrm{H}_{2}\right) \mathrm{ppm} .{ }^{13} \mathrm{C}\left\{{ }^{1} \mathrm{H}\right\}$ $\operatorname{NMR}\left(\mathrm{C}_{6} \mathrm{D}_{6}\right): \delta 20.06,22.04,27.60,41.91,108.84,122.59,126.16,129.48,130.67,139.79 \mathrm{ppm}$. Anal. Calc. $\left(\mathrm{C}_{10} \mathrm{H}_{12} \mathrm{BrN}\right)$ : C, 53.12; H, 5.35; N, 6.19\%. Found: C, 53.30; H, 5.13; N, $6.51 \%$.

Compound 10. The compound was synthesized by the same conditions and procedures as for 5a using 9. It was purified by column chromatography on silica gel eluting with hexane and ethyl acetate (2 : 1). A light yellow solid was obtained (90\%). M.p. 67-69 ${ }^{\circ} \mathrm{C}$. IR (neat): $3375(\mathrm{~N}-\mathrm{H}), 1700(\mathrm{C}=\mathrm{O})$ 
$\mathrm{cm}^{-1} .{ }^{1} \mathrm{H}$ NMR $\left(\mathrm{C}_{6} \mathrm{D}_{6}\right): \delta 0.77\left(\mathrm{~d}, J=7.2 \mathrm{~Hz}, 3 \mathrm{H}, \mathrm{CH}_{3}\right), 1.59-1.70\left(\mathrm{~m}, 2 \mathrm{H}, \mathrm{CH}_{2} \mathrm{CH}_{2} \mathrm{CH}_{2}\right), 1.65(\mathrm{~s}, 3 \mathrm{H}$, $\left.\mathrm{CH}_{3}\right), 1.84\left(\mathrm{dd}, J=2.4,18.4 \mathrm{~Hz}, 1 \mathrm{H}, \mathrm{OCCH}_{2}\right), 2.21\left(\mathrm{~s}, 3 \mathrm{H}, \mathrm{CH}_{3}\right), 2.20-2.30(\mathrm{~m}, 1 \mathrm{H}, \mathrm{CH}), 2.44$ (dd, $J=$ 6.4, $18.4 \mathrm{~Hz}, 1 \mathrm{H}, \mathrm{OCCH}_{2}$ ), 2.60 (br t, $J=6 \mathrm{~Hz}, 2 \mathrm{H}, \mathrm{CH}_{2}$ ), 2.97 (br t, $J=5.6 \mathrm{~Hz}, 2 \mathrm{H}, \mathrm{N}-\mathrm{CH}_{2}$ ), 4.06 (s, $1 \mathrm{H}, \mathrm{NH}), 6.66\left(\mathrm{~s}, 1 \mathrm{H}, \mathrm{CH}, \mathrm{C}_{6} \mathrm{H}_{2}\right), 6.74\left(\mathrm{~s}, 1 \mathrm{H}, \mathrm{C}_{6} \mathrm{H}_{2}\right) \mathrm{ppm} .{ }^{13} \mathrm{C}\left\{{ }^{1} \mathrm{H}\right\} \operatorname{NMR}\left(\mathrm{C}_{6} \mathrm{D}_{6}\right): \delta 15.83,19.06,20.58$, 22.51, 27.92, 37.52, 42.48, 43.55 ppm. Anal. Calc. $\left(\mathrm{C}_{17} \mathrm{H}_{21} \mathrm{NO}\right)$ : C, 79.96; H, 8.29; N, 5.49 \%. Found: C, $80.17 ; \mathrm{H}, 8.44 ; \mathrm{N}, 5.75 \%$.

Compound 11. The compound was synthesized by the similar conditions and procedures as for $\mathbf{6 a}$ using 10. $\mathrm{La}(\mathrm{OTf})_{3}$ was used instead of $\mathrm{CeCl}_{3}$. Light yellow solid was obtained in $42 \%$ yield. ${ }^{1} \mathrm{H}$ NMR $\left(\mathrm{C}_{6} \mathrm{D}_{6}\right): \delta 1.66-1.71\left(\mathrm{~m}, 2 \mathrm{H}, \mathrm{CH}_{2} \mathrm{CH}_{2} \mathrm{CH}_{2}\right), 1.80\left(\mathrm{~s}, 3 \mathrm{H}, \mathrm{CH}_{3}\right), 1.89\left(\mathrm{~s}, 3 \mathrm{H}, \mathrm{CH}_{3}\right), 1.90\left(\mathrm{~s}, 3 \mathrm{H}, \mathrm{CH}_{3}\right), 2.24$ (s, 3H, $\mathrm{CH}_{3}$ ), 2.64 (br t, $\left.J=6.4 \mathrm{~Hz}, 2 \mathrm{H}, \mathrm{CH}_{2}\right), 2.74$ (d, $\left.J=2 \mathrm{~Hz}, 2 \mathrm{H}, \mathrm{CH}_{2}\right), 2.86-2.92\left(\mathrm{~m}, 2 \mathrm{H}, \mathrm{N}-\mathrm{CH}_{2}\right)$, 3.62 (br s, $1 \mathrm{H}, \mathrm{NH}), 6.75\left(\mathrm{~s}, 1 \mathrm{H}, \mathrm{C}_{6} \mathrm{H}_{2}\right), 6.77\left(\mathrm{~s}, 1 \mathrm{H}, \mathrm{C}_{6} \mathrm{H}_{2}\right) \mathrm{ppm} .{ }^{13} \mathrm{C}\left\{{ }^{1} \mathrm{H}\right\} \mathrm{NMR}\left(\mathrm{C}_{6} \mathrm{D}_{6}\right): \delta 11.85,13.61$, $14.39,20.74,22.86,27.70,42.20,48.88,120.81,122.01,124.78,128.68,129.36,132.87,136.36$, $136.65,140.75,141.15 \mathrm{ppm}$.

Complex 8. It was synthesized by the same conditions and procedures as for 7a using 20. It was purified by trituration in pentane. Overall yield from 20 was $63 \%$. The ${ }^{1} \mathrm{H}$ NMR datum for the intermediate bis(dimethylamido)titanium complex: ${ }^{1} \mathrm{H}$ NMR $\left(\mathrm{C}_{6} \mathrm{D}_{6}\right): \delta 1.69-1.74\left(\mathrm{~m}, 2 \mathrm{H}, \mathrm{CH}_{2} \mathrm{CH}_{2} \mathrm{CH}_{2}\right)$, $1.86\left(\mathrm{~s}, 3 \mathrm{H}, \mathrm{CH}_{3}\right), 1.88\left(\mathrm{~s}, 3 \mathrm{H}, \mathrm{CH}_{3}\right), 1.92\left(\mathrm{~s}, 3 \mathrm{H}, \mathrm{CH}_{3}\right), 2.31\left(\mathrm{~s}, 3 \mathrm{H}, \mathrm{CH}_{3}\right), 2.57\left(\mathrm{t}, J=5.6 \mathrm{~Hz}, 2 \mathrm{H}, \mathrm{CH}_{2}\right)$, $2.95\left(\mathrm{~s}, 6 \mathrm{H}, \mathrm{NCH}_{3}\right), 3.27\left(\mathrm{~s}, 6 \mathrm{H}, \mathrm{NCH}_{3}\right), 4.02\left(\mathrm{ddd}, J=5.2,7.2,12.0 \mathrm{~Hz}, 1 \mathrm{H}, \mathrm{NCH}_{2}\right), 4.24(\mathrm{dt}, J=5.2$, 12.4Hz, $\left.1 \mathrm{H}, \mathrm{NCH}_{2}\right), 5.78(\mathrm{~s}, 1 \mathrm{H}, \mathrm{Cp}-\mathrm{H}), 6.77\left(\mathrm{~s}, 1 \mathrm{H}, \mathrm{C}_{6} \mathrm{H}_{2}\right), 6.91\left(\mathrm{~s}, 1 \mathrm{H}, \mathrm{C}_{6} \mathrm{H}_{2}\right) \mathrm{ppm}$. The analytical data for 10: ${ }^{1} \mathrm{H}$ NMR $\left(\mathrm{C}_{6} \mathrm{D}_{6}\right)$ : $\delta 1.36-1.44\left(\mathrm{~m}, 2 \mathrm{H}, \mathrm{CH}_{2} \mathrm{CH}_{2} \mathrm{CH}_{2}\right), 1.76\left(\mathrm{~s}, 3 \mathrm{H}, \mathrm{CH}_{3}\right), 1.85\left(\mathrm{~s}, 3 \mathrm{H}, \mathrm{CH}_{3}\right), 2.07$ (s, 3H, $\left.\mathrm{CH}_{3}\right), 2.18\left(\mathrm{~s}, 3 \mathrm{H}, \mathrm{CH}_{3}\right), 2.12\left(\mathrm{t}, J=4 \mathrm{~Hz}, 2 \mathrm{H}, \mathrm{CH}_{2}\right), 4.50-4.70\left(\mathrm{~m}, 2 \mathrm{H}, \mathrm{N}-\mathrm{CH}_{2}\right), 6.02(\mathrm{~s}, 1 \mathrm{H}, \mathrm{Cp}-$ $\mathrm{H}), 6.59\left(\mathrm{~s}, 1 \mathrm{H}, \mathrm{C}_{6} \mathrm{H}_{2}\right), 6.78\left(\mathrm{~s}, 1 \mathrm{H}, \mathrm{C}_{6} \mathrm{H}_{2}\right) \mathrm{ppm} .{ }^{13} \mathrm{C}\left\{{ }^{1} \mathrm{H}\right\} \mathrm{NMR}\left(\mathrm{C}_{6} \mathrm{D}_{6}\right): \delta 12.76,14.87,15.06,21.14$, 22.39, 26.32, 54.18, 117.49, 120.40, 126.98, 129.53, 130.96, 131.05, 133.19, 143.22, 143.60, 160.82 ppm. Anal. Calc. $\left(\mathrm{C}_{18} \mathrm{H}_{21} \mathrm{Cl}_{2} \mathrm{NTi}\right)$ : C, 58.41; H, 5.72; N, 3.78\%. Found: C, 58.19; H, 5.93; N, $3.89 \%$.

Polymerizations. Inside a dry box, to a dried $70 \mathrm{~mL}$ glass reactor was added $30 \mathrm{~mL}$ of toluene solution of 1-hexene $(0.30 \mathrm{M})$. The reactor was assembled and brought out from the dry box. The reactor was heated to $90{ }^{\circ} \mathrm{C}$ by mantle. After an activated catalyst, which was prepared by mixing the complex $(0.50 \mu \mathrm{mol} \mathrm{Ti}),\left[\mathrm{CPh}_{3}\right]^{+}\left[\mathrm{B}\left(\mathrm{C}_{6} \mathrm{~F}_{5}\right)_{4}\right]^{-}(2.0 \mu \mathrm{mol})$, and $(\mathrm{iBu})_{3} \mathrm{Al}(0.200 \mathrm{mmol})$ for 5 minutes, was added via a syringe, ethylene (100 psig) was fed immediately. After polymerization was conducted for 5 minutes, the ethylene was vented and the acetone was added to the reactor to give white precipitates which were collected by filtration and dried under vacuum at $88-100{ }^{\circ} \mathrm{C}$ for 5 hours. In the ${ }^{1} \mathrm{H}$ NMR spectra of the copolymers, the methyl $\left(\mathrm{CH}_{3}\right)$ signals $(0.93-1.02 \mathrm{ppm})$ was well isolated from the methine $(\mathrm{CH})$ and methylene $\left(\mathrm{CH}_{2}\right)$ signals $(1.30-1.50 \mathrm{ppm})$ and the 1-hexene contents were calculated from the 
integration values of the methyl signal and the combined signal of methine and methylene (See the typical ${ }^{1} \mathrm{H}$ NMR spectrum of the copolymer below). The copolymer $(10 \mathrm{mg})$ was dissolved in $\mathrm{C}_{6} \mathrm{D}_{6}$ and the ${ }^{1} \mathrm{H}$ NMR spectra were obtained at $78{ }^{\circ} \mathrm{C}$. The copolymer $(20 \mathrm{mg})$ was dissolved in $\mathrm{C}_{6} \mathrm{D}_{6}$ and the ${ }^{13} \mathrm{C}$ NMR spectrum was obtained $\left(100 \mathrm{MHz}, 90^{\circ}\right.$ pulse angle, 10 second relaxation delay time, 4000 acquisition number). The 1-hexene content calculated roughly from the ${ }^{13} \mathrm{C}$ NMR spectrum ${ }^{6}$ was in agreement with that calculated from the ${ }^{1} \mathrm{H}$ NMR spectrum.
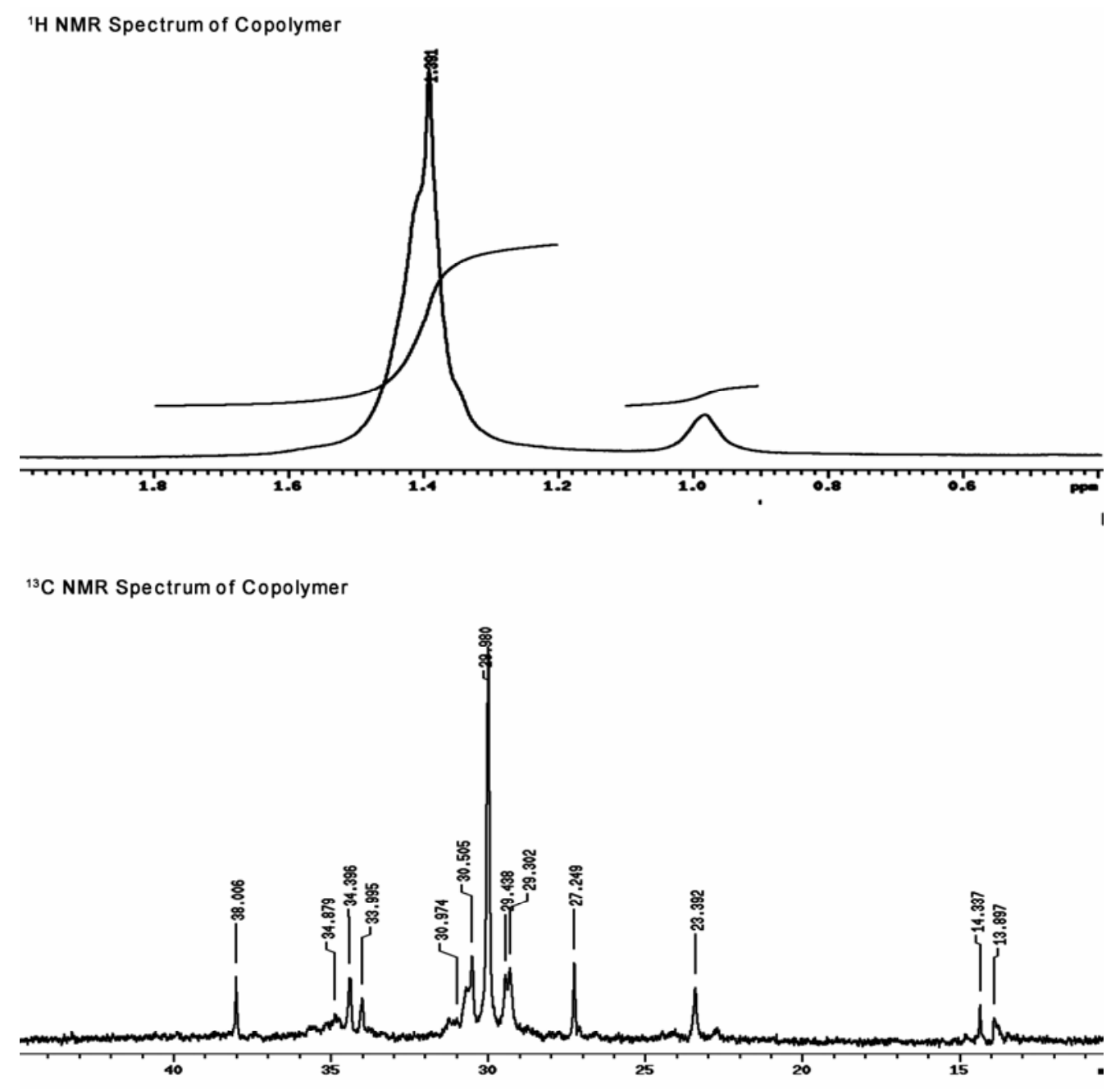

Crystallographic Studies. Crystals of 7a and 7d were mounted in thin-walled glass capillaries and sealed under argon. The data sets were collected on a Bruker Smart CCD detector single diffractometer. Mo-K $\alpha$ radiation $(\lambda=0.7107 \AA$ ) was used for all structures. Structure of $7 \mathbf{a}$ was solved by the application of Patterson method and the structure of $\mathbf{7 d}$ was solved by the direct methods using the SHELX-96 program and least-squares refinement using the SHELXL-Plus (5.1) software package. All 
non-hydrogen atoms were refined anisotropically. All hydrogen atoms were included in the calculated positions. The crystal data and refinement results are summarized below in Table 1 .

Table 1. Crystallographic Parameters of 7a and 7d

\begin{tabular}{|c|c|c|}
\hline & $7 a$ & $7 d$ \\
\hline formula & {$\left[\mathrm{C}_{20} \mathrm{H}_{25} \mathrm{Cl}_{2} \mathrm{NTi}\right]_{2}$} & $\mathrm{C}_{20} \mathrm{H}_{24} \mathrm{Cl}_{3} \mathrm{NTi}$ \\
\hline Fw & 796.42 & 432.65 \\
\hline color & red & red \\
\hline size, $\mathrm{mm}^{3}$ & $0.42 \times 0.29 \times 0.19$ & $0.33 \times 0.24 \times 0.06$ \\
\hline$a, \AA$ & $8.7730(18)$ & $8.3533(10)$ \\
\hline$b, \AA$ & $13.301(3)$ & $11.2763(13)$ \\
\hline$c, \AA$ & $17.470(4)$ & $11.9066(14)$ \\
\hline$\alpha, \operatorname{deg}$ & $82.526(4)$ & $99.884(2)$ \\
\hline$\beta, \operatorname{deg}$ & $82.582(4)$ & $98.641(2)$ \\
\hline$\gamma, \operatorname{deg}$ & $87.424(4)$ & $106.940(2)$ \\
\hline $\mathrm{V}, \AA^{3}$ & $2003.6(7)$ & $1032.8(2)$ \\
\hline crystal system & triclinic & triclinic \\
\hline space group & $\mathrm{P}-1$ & $\mathrm{P}-1$ \\
\hline$D($ calc $), \mathrm{gcm}^{-1}$ & 1.320 & 1.391 \\
\hline$Z$ & 2 & 2 \\
\hline$\mu, \mathrm{mm}^{-1}$ & 0.695 & 0.805 \\
\hline no. of data collected & 27027 & 13924 \\
\hline no. of unique data & 9883 & 5066 \\
\hline no. of variables & 439 & 229 \\
\hline $\mathrm{R}(\%)$ & 0.0336 & 0.0607 \\
\hline $\mathrm{R}_{\mathrm{w}}(\%)$ & 0.0890 & 0.2040 \\
\hline Goodness of fit & 1.046 & 1.134 \\
\hline
\end{tabular}


${ }^{\mathrm{a}}$ Data collected at 293(2) $\mathrm{K}$ with Mo-K $\alpha$ radiation $(\lambda(\mathrm{K} \alpha)=0.7107 \AA), \mathrm{R}(F)=\Sigma|| F_{\mathrm{o}}|-| F_{\mathrm{c}}|/ \Sigma| F_{\mathrm{o}} \mid$ with $F_{\mathrm{o}}>2.0 \sigma(I), \mathrm{R}_{\mathrm{w}}=\left[\Sigma\left[w\left(F_{\mathrm{o}}^{2}-F_{\mathrm{c}}^{2}\right)^{2}\right] / \Sigma\left[w\left(F_{\mathrm{o}}\right)^{2}\right]^{2}\right]^{1 / 2}$ with $F_{\mathrm{o}}>2.0 \sigma(I)$.

${ }^{1}$ Conia, J. M.; Leriverend, M. L. Bull. Soc. Chim. Fr. 1970, 2981.

${ }^{2}$ Gage, J. R.; Wagner, J. M. J. Org. Chem, 1995, 60, 2613.

${ }^{3}$ Ma, H.; Shen, Y.-Q.; Chen, P.; Wang, Y.-M. Meng, J.-B. Eur. Polym. J. 1998, 34, 1125.

${ }^{4}$ Kasashima, Y.; Kumada, H.; Yamamoto, K.; Akutsu, F.; Naruchi, K.; Miura, M. Polymer 1995, 36, 645.

${ }^{5}$ Marchand, P.; Puget, A.; Le Baut, G.; Emig, P.; Czech, M.; Günther, E. Tetrahedron 2005, 61, 4034.

${ }^{6}$ Cheng, H. N. Polym. Bull. 1991, 26, 325. 